\title{
Nonlinear Stability and instability of relativistic Vlasov-Maxwell systems
}

\author{
Zhiwu Lin \\ Courant Institute \\ Walter Strauss \\ Brown University
}

\begin{abstract}
We prove the nonlinear stability or instability of certain periodic equilibria of the $1 \frac{1}{2} \mathrm{D}$ relativistic Vlasov-Maxwell system. In particular, for a purely magnetic equilibrium with vanishing electric field, we prove its nonlinear stability under a sharp criterion by extending the usual Casimir-energy method in several new ways. For a general electromagnetic equilibrium we prove that nonlinear instability follows from linear instability. The nonlinear instability is macroscopic, involving only the $L^{1}$-norms of the electromagnetic fields.
\end{abstract}

(c) 2000 Wiley Periodicals, Inc.

\section{Introduction}

In this paper, we prove the true nonlinear stability or instability of certain equilibria of the $1 \frac{1}{2} \mathrm{D}$ relativistic Vlasov-Maxwell (RVM) system. We use the same notation as in [15]. The $1 \frac{1}{2} \mathrm{D}$ RVM system of two species of particles without external fields is

$$
\partial_{t} f^{ \pm}+\hat{v}_{1} \partial_{x} f^{ \pm} \pm\left(E_{1}+\hat{v}_{2} B\right) \partial_{v_{1}} f^{ \pm} \pm\left(E_{2}-\hat{v}_{1} B\right) \partial_{v_{2}} f^{ \pm}=0
$$

$$
\partial_{t} E_{1}=-j_{1}, \quad \partial_{t} E_{2}+\partial_{x} B=-j_{2}
$$

with

$$
\partial_{t} B=-\partial_{x} E_{2}, \quad \partial_{x} E_{1}=\rho
$$

$$
\rho=\int\left(f^{+}-f^{-}\right) d v, \quad j_{i}=\int \hat{v}_{i}\left(f^{+}-f^{-}\right) d v(i=1,2) .
$$

We refer to [15] for further discussion of this model. Consider a $P$-periodic equilibrium of the form

$$
f^{0, \pm}=\mu^{ \pm}\left(e^{ \pm}, p^{ \pm}\right)=\mu^{ \pm}\left(\langle v\rangle \pm \phi^{0}(x), v_{2} \pm \psi^{0}(x)\right) \geq 0
$$

with

$$
E_{1}^{0}=-\partial_{x} \phi^{0}, \quad E_{2}^{0}=0, \quad B^{0}=\partial_{x} \psi^{0}
$$


where $\left(\phi^{0}, \psi^{0}\right)$ satisfy the ODE system

(1.3)

$$
\partial_{x}^{2} \phi^{0}=-\rho^{0}=-\int\left(f^{0,+}-f^{0,-}\right) d v, \quad \partial_{x}^{2} \psi^{0}=-j_{2}^{0}=-\int \hat{v}_{2}\left(f^{0,+}-f^{0,-}\right) d v .
$$

In the appendix of [15], we showed that there exist infinitely many periodic electromagnetic equilibria of this form. Under the assumption $\mu_{e}^{ \pm}<0$, we found in [15] a nearly sharp linear stability criterion for periodic equilibria of the form (1.2). Various linearly stable and unstable examples were explicitly constructed using this criterion. The main purpose of the present paper is to prove that these linear stability or instability criteria are indeed also true on the nonlinear dynamical level at least in some cases.

First we show that the existence of a growing mode implies nonlinear instability, in the precise sense of the following theorem.

Theorem 1.1. (Instability) Let $\left(f^{0, \pm}=\mu^{ \pm}\left(e^{ \pm}, p^{ \pm}\right), E^{0}, B^{0}\right)$ be a periodic equilibrium of the form (1.2) for system (1.1c) with

$$
\mu^{ \pm}=O\left(\langle v\rangle^{-\eta}\right), \eta>3,\left|\mu_{e}^{ \pm}\right|+\left|\mu_{p}^{ \pm}\right|=O\left(\mu^{ \pm}\right), \sup _{x} \int\langle v\rangle^{2}\left(\left|\mu_{e}^{ \pm}\right|+\left|\mu_{p}^{ \pm}\right|\right) d v<\infty .
$$

If there exists a growing mode, then there exist positive constants $\varepsilon_{0}, C_{1}$ and a family of $W^{1,1}$ solutions $\left[\bar{f}^{\delta, \pm}, \bar{E}^{\delta}, \bar{B}^{\delta}\right]$ of $(1.1 c)$ with period $P$ in $x$, defined for $\delta$ sufficiently small, with $\bar{f}^{\delta, \pm}(t)$ non-negative, such that

$$
\left\|\bar{f}^{\delta, \pm}(0)-f^{0}\right\|_{W^{1,1}}+\left\|\bar{E}^{\delta}(0)-E^{0}\right\|_{W^{1,1}}+\left\|\bar{B}^{\delta}(0)-B^{0}\right\|_{W^{1,1}} \leq \delta
$$

and

$$
\sup _{0 \leq t \leq C_{1}|\ln \delta|}\left\|\bar{E}_{1}^{\delta}(t)-E_{1}^{0}\right\|_{L^{1}}+\left\|\bar{E}_{2}^{\delta}(t)\right\|_{L^{1}}+\left\|\bar{B}^{\delta}(t)-B^{0}\right\|_{L^{1}} \geq \varepsilon_{0} .
$$

We emphasize that the conclusion of nonlinear instability proven in Theorem 1.1 is in the macroscopic sense: the fields themselves deviate at some time from the equilibrium fields. This is physically natural since it excludes the spurious instability due to microscopic oscillations of the distribution function $f$. In [9] nonlinear instability was proven for homogeneous equilibria in a norm that included the $L^{1}$ deviation of the distribution function $f$. So our result is an improvement of [9] in two ways: the instability is proven more generally for any inhomogeneous equilibrium, and it is proven in a stronger, more physical sense.

Secondly, we prove nonlinear stability, for certain purely magnetic equilibria of the type

$$
f^{0, \pm}=\mu^{ \pm}\left(e, p^{ \pm}\right)=\mu^{ \pm}\left(\langle v\rangle, v_{2} \pm \psi^{0}(x)\right), B^{0}=\partial_{x} \psi^{0}, E^{0}=0 .
$$

Such equilibria exist ([15]) if we assume that $\mu^{+}(e, p)=\mu^{-}(e,-p)$ and $\psi^{0}$ satisfies the ODE

$$
\partial_{x}^{2} \psi^{0}=2 \int \hat{v}_{2} \mu^{-}\left(\langle v\rangle, v_{2}-\psi^{0}(x)\right) d v
$$


Let $T_{\psi^{0}}$ be the minimal period of the periodic solution $\psi^{0}$ to (1.4). By adjusting its starting point, we can always arrange that the solution satisfies

$$
\begin{gathered}
\psi^{0}(0)=\psi^{0}\left(T_{\psi^{0}}\right)=\min _{0 \leq x \leq T_{\psi^{0}}} \psi^{0}(x), \quad \psi^{0}\left(\frac{1}{2} T_{\psi^{0}}\right)=\max _{0 \leq x \leq T_{\psi^{0}}} \psi^{0}(x), \\
\psi^{0}(x)=\psi^{0}\left(T_{\psi^{0}}-x\right), \quad \forall x \in\left[0, T_{\psi^{0}}\right],
\end{gathered}
$$

and $\psi^{0}(x)$ is strictly increasing in the interval $\left[0, \frac{1}{2} T_{\psi^{0}}\right]$. Assume $\mu_{e}^{ \pm}<0$. Moreover, we consider general perturbations of period $T_{\psi^{0}}$. As noted in [15], the equilibrium is more likely to be unstable under perturbations that have periods that are multiples of $T_{\psi^{0}}$. Given such an equilibrium, we proved in [15] the sharp linear stability criterion for perturbations of period $T_{\psi^{0}}$, namely, that the operator

$$
\mathscr{L}^{0} h=-\partial_{x}^{2} h-2 \int \hat{v}_{2} \mu_{p}^{-} d v h-2 \int \hat{v}_{2} \mu_{e}^{-} \mathscr{P}^{-}\left[\hat{v}_{2} h\right] d v
$$

(which has discrete spectrum) should be nonnegative, where $\mathscr{P}^{-}$is defined as the projection operator from $L_{\left|\mu_{e}^{-}\right|}^{2}$ to $\operatorname{ker} D^{-}$with $D^{-}=\hat{v}_{1} \partial_{x}-\hat{v}_{2} B^{0} \partial_{v_{1}}+\hat{v}_{1} B^{0} \partial_{v_{2}}$ and $L_{\left|\mu_{e}^{-}\right|}^{2}$ denotes the $\left|\mu_{e}^{-}\right|$-weighted $L_{x, v}^{2}$ space. Using this stability criterion, we constructed an inhomogeneous equilibrium in [15] that is linearly stable. We emphasize that the nonlocal stabilizing term involving $\mathscr{P}^{-}$is indispensable to a full stability analysis, even for a homogeneous equilibrium.

In the present paper we show that $\mathscr{L}^{0} \geq 0$ also implies nonlinear stability, at least in certain cases. We restrict our attention to purely magnetic equilibria of the special type

$$
f^{0, \pm}(x, v)=\mu^{ \pm}\left(e, p^{ \pm}\right)=e^{-\langle v\rangle} \gamma\left(v_{2} \pm \psi^{0}(x)\right), B^{0}=\partial_{x} \psi^{0}(x), E^{0}=0
$$

where $\gamma \in C^{3}$ is even. Our reason for this specialization is mainly for simplicity. For such equilibria many calculations can be made explicit without being excessively technical. We do expect that our ideas should carry over to prove the nonlinear stability of more general purely magnetic equilibria.

Theorem 1.2. (Stability) Consider a purely magnetic equilibrium of the form (1.6). Let $\eta=\log \gamma$ and assume $\eta^{\prime}, \eta^{\prime \prime}, \eta^{\prime \prime \prime}$ are bounded. If $\mathscr{L}^{0} \geq 0$ and $\operatorname{ker} \mathscr{L}^{0}$ is onedimensional, then the equilibrium is nonlinearly stable in the following sense. Given $\varepsilon>0$, there exists $\delta>0$ with the following property. Let initial data which launches a global solution be given with $x$-period $P=T_{\psi^{0}}$, which satisfies $\int_{0}^{P} B(0) d x=0$ and $\partial_{x} E_{1}(0)=\rho(0)$, and for which

$$
d\left(f^{ \pm}(0)-\mu^{ \pm}, \psi(0)-\psi^{0}, E\right)<\delta,
$$

where the distance functional $d\left(f^{ \pm}-\mu^{ \pm}, \psi-\psi^{0}, E\right)$ is defined by (2.7) below. Then the solution of $(1.1 c)$ satisfies

$$
\inf _{\theta} d\left(T_{\theta} f^{ \pm}(t)-\mu^{ \pm}, T_{\theta} \psi(t)-\psi^{0}, T_{\theta} E\right)<\varepsilon
$$


at all later times $0<t<\infty$, where $T_{\theta}$ denotes the translation in $x$ by $\theta$. In particular, it satisfies

$$
\sup _{0<t<\infty} \inf _{\theta}\left\{\left\|T_{\theta} f^{ \pm}(t)-\mu^{ \pm}\right\|_{L^{1}}+\left\|T_{\theta} B(t)-B^{0}\right\|_{L^{2}}+\left\|T_{\theta} E\right\|_{L^{2}}\right\}<\varepsilon .
$$

Sufficient conditions for initial conditions to launch a solution of (1.1c) are $f(0) \in B V ;\langle v\rangle^{3+\alpha} f(0) \in L^{\infty} ; E(0), B(0) \in W^{1, \infty}$ (see Theorem 4 of [8]).

We remark that Theorem 1.2 and its proof go over almost verbatim to the $1 \frac{1}{2} \mathrm{D}$ RVM system on the whole line rather than on the circle. For purely magnetic solitary wave and kink-type solutions on the whole line, we can obtain the sharp nonlinear stability criterion as above; that is, $\mathscr{L}^{0} \geq 0$ and $\operatorname{ker} \mathscr{L}^{0}=\left\{\psi_{0 x}\right\}$ imply nonlinear stability with $\mathscr{L}^{0}$ defined by (1.5) on the whole line.

In [6], Guo proved the nonlinear stability of purely magnetic equilibria of the same form (1.6) in the periodic and the whole line cases. The nonlinear stability criterion proven in [6] was that the operator

$$
L=-\partial_{x}^{2}-2 \int \hat{v}_{2} \mu_{p}^{-} d v
$$

should be nonnegative and $\operatorname{ker} L$ should be one-dimensional. For a kink-type equilibrium, one has $L \geq 0$ and the nonlinear stability can be proven. However, for periodic and solitary wave type equilibria, since $L \psi_{x}^{0}=0$ and $\psi_{x}^{0}$ has a zero, the operator $L$ must have a negative eigenvalue by Sturm-Liouville theory. So the stability criterion $L \geq 0$ could never be satisfied for periodic or solitary wave type equilibria. Thus in [6] the periodic waves were only proven to be conditionally stable under perturbations for which the magnetic field $B$ is an even function and $L$ is a nonnegative operator. For the same reason, the nonlinear stability of homogeneous equilibria claimed in [9] is also conditional, with the evenness of $B$ implicitly assumed for the perturbations. On the other hand, in [15], the homogeneous equilibria studied in [9] are proven to be linearly stable without any restriction on the parity of the perturbations and we construct stable inhomogeneous examples. The proofs crucially require the additional stabilizing term $-2 \int \hat{v}_{2} \mu_{e}^{-} \mathscr{P}^{-}\left[\hat{v}_{2} h\right] d v$ in $\mathscr{L}^{0}$. By Theorem 1.2, these linearly stable equilibria are also nonlinearly stable. Now we describe the main ideas in the proof of Theorems 1.1 and 1.2.

To prove nonlinear instability for Vlasov systems, one has to overcome several particular difficulties. The first difficulty is that the nonlinear term in the Vlasov equation (1.1a) contains $\partial_{v} f$. To overcome this difficulty of a "loss of derivative", Guo and Strauss introduced a bootstrap technique in a series of papers starting with [7]. This allowed them to get a growth estimate of $\partial_{v} f$ from the growth of $f$ itself, within a time period $\left[0, T_{\delta}\right]$ during which the perturbation is exponentially growing while the amplitude is kept smaller than $\delta$. They close the estimate by showing that the nonlinear term is indeed of higher order in $\left[0, T_{\delta}\right]$, which implies the nonlinear instability. 
A less obvious difficulty is related to the positive Liapunov exponent (growth rate) $\mu$ of the particle trajectory in steady fields. It turns out that when $\mu$ is less than the growth rate $\lambda$ of the full linearized RVM system, the growing mode is not differentiable, and more seriously the bootstrap estimate of $\partial_{v} f$ cannot be obtained in this case. For the weakly inhomogeneous ([7]) and homogeneous ([9]) cases, one has the "good" case since $\lambda>\mu$. However for general inhomogeneous equilibria, the "bad case" when $\lambda<\mu$ has to be dealt with. In the proof of nonlinear instability of the 1D Vlasov-Poisson system, Z. Lin ([12]) introduced two new ideas to surmount these difficulties. One was to estimate the electric field $E$ only, thus utilizing the regularizing effect of going from $f$ to $E$ by the Poisson equation in order to balance the derivative $\partial_{v}$ in the nonlinear term of the Vlasov equation. This overcomes the "loss of derivative".

The other new idea in [12] was to replace the classical Liapunov exponent $\mu$ by an averaging Liapunov exponent $\mu_{a v}$. This comes from a rather delicate analysis of the geometric properties of the particle trajectory. Coupled with the easier bootstrap estimates involving no derivative of $f$, these new ideas led to the nonlinear instability result ([12]) in the electric field.

In this paper, we generalize these ideas to the relativistic Vlasov-Maxwell system to prove Theorem 1.1. However, there are still some important differences from all the earlier papers. First, unlike the Vlasov-Poisson case one does not gain any obvious regularity by passing from the particle density $f$ to the field $E, B$ since the Maxwell system is hyperbolic. To overcome this difficulty, we use the operator splitting ideas introduced in [4] (for 3D) and [5] (for $1 \frac{1}{2} \mathrm{D}$ ). This allows us to gain some regularity when the particle speed is finite, in which case the characteristics of Vlasov and Maxwell equations are strictly separated. To deal with particles of arbitrarily high speed, we estimate the coefficients coming from the operator splitting by a power of $\langle v\rangle$. This allows us to obtain some regularity of $E$ and $B$ by using a $\langle v\rangle^{k}$-weighted norm for $f$. It turns out that this regularity is enough to overcome the "loss of derivative" by using a duality argument. Secondly, we also need to introduce the averaging Liapunov exponent and prove that it vanishes. The proof is more involved than in the Vlasov-Poisson case since the particle trajectory in electromagnetic fields is much more complicated than in electrostatic fields. Here the result in [13] for ideal plane flows is important. The main line of proof of Theorem 1.1 follows that of [12] but is technically much more involved. The estimate of the linearized semigroup and the bootstrap estimates are really different from and more difficult than the Vlasov-Poisson case. Several techniques regarding homogenous equilibria ([9]) are also very useful in our study of inhomogeneous equilibria.

In order to prove the nonlinear stability result of Theorem 1.2, we also introduce several new ideas. First, let us make some general comments about the usual nonlinear stability proofs. The usual way of proving nonlinear stability is the socalled energy-Casimir method. This idea was first introduced by Newcomb (see the appendix of [2]) and used by Gardner [3] for Vlasov plasmas and then by Arnold 
[1] for ideal plane flows. This method has been used extensively since then in the analysis of nonlinear stability in fluid and plasmas (e.g. [10]). The idea is very simple: one constructs an energy-Casimir functional that is an invariant of the nonlinear system under consideration. Its first variation vanishes at the equilibrium. Performing a Taylor expansion of the functional around the equilibrium, one then tries to prove that the second-order term is a positive quadratic form which is to be used as the nonlinear stability norm.

To close the argument, one has to show that the remainder term in the Taylor expansion is of higher order (smaller) in the stability norm. In Vlasov models one of the remainder terms is $f^{3}$ whose $L^{2}$ norm is difficult to be bounded by a power of the stability norm. In the earlier papers using energy-Casimir methods ([1], [10]), the nonlinear term is estimated by a convexity argument and the higherorder estimate is avoided. However, the stability condition obtained in this simple way is far from being sharp and is not even applicable to many situations like our current case.

To get a sharper stability criterion, one has to use the full power of the positive quadratic term and to carefully estimate the higher order term. In [6] for $1 \frac{1}{2} \mathrm{D}$ RVM, a delicate argument was developed to get the higher order estimate and the sufficient stability criterion $L>0$ (with $L$ defined in (1.8)) was derived. But, as mentioned before, for the periodic and solitary wave cases, the operator $L$ always has a negative eigenvalue and thus general nonlinear stability cannot be proven by this stability criterion. To get the sharp nonlinear stability criterion in Theorem 1.2, one has to extend the energy-Casimir method in several important ways.

To understand these ideas better, we switch for a moment to the simpler case of ideal plane flows. In [16], Wolansky and Ghil extended the energy-Casimir method for ideal plane flows in several directions. They used any finite number of Casimir functionals as constraints, thus constructing an augmented energy-Casimir functional with Lagrange multipliers. Doing a Taylor expansion of this augmented functional, they obtained a sharper estimate for the quadratic term. The way they handled the nonlinear term is also interesting. By a duality argument using a Legendre transformation, they essentially transformed the nonlinear term for the vorticity to the one for the stream function. This new nonlinear term was easily shown to be of higher order. Combining these ideas, they were able to obtain a nonlinear stability criterion which is nonlocal since a finite-dimensional projection is involved. In [11], by using all the Casimir functionals as constraints, a sharper and more explicit criterion was obtained. However, as pointed out in [11], there is a strict gap between the nonlinear stability criterion thereby obtained and the apparently sharp spectral stability criterion. This gap occurs because the particle with fixed energy in the steady flow is usually trapped on two or more disjoint closed curves. To get a sharp nonlinear stability criterion, or to pass from linear stability to nonlinear stability, one has to close this gap. 
We now return to $1 \frac{1}{2} \mathrm{D}$ RVM. The proof of Theorem 1.2 is accomplished in several steps and several new ideas and techniques are introduced. First, we deduce a nonlinear stability criterion by Taylor-expanding the usual energy-Casimir functional (2.1) using all the Casimir functionals as constraints. More precisely, by observing that all of the functionals $I_{g}\left(f^{ \pm}, v_{2} \pm \psi\right)=\int g\left(f^{ \pm}, v_{2} \pm \psi\right) d v d x$ are invariant, the conditions $I_{g}\left(f^{ \pm}, v_{2} \pm \psi\right)=I_{g}\left(\mu^{ \pm}, v_{2} \pm \psi^{0}\right)$ impose additional constraints on the perturbations. In a similar fashion to the ideal plane flow case in [11], we only need to incorporate finitely many Casimir constraints. However, unlike the case of ideal plane flows in [16], we are not able to construct a similar augmented functional to utilize these constraints in the plasma case. This difficulty occurs in part because $f$ and $\psi$ are functions living in different spaces. More significantly, in the plasma case $f$ cannot be represented by $\psi$ via a local relationship. In the present paper, in order to use the Casimir constraints, we instead employ a new technique originally developed for ideal plane flows in [14]. In [11] and [14] the idea is to directly minimize the difference of the energy-Casimir functional under the Casimir constraints. The constraints are incorporated by using the corresponding finite-dimensional projection for the vorticity $\omega$. The nonlinear term in $\omega$ is transformed to a new one in the stream function $\varphi$ by the Legendre transformation as in [16]. The key observation is that the constraints on $\omega$ in the projection form are nicely suited to the Legendre transformation and provide a nonlinear nonlocal term in $\varphi$ involving the projection. Performing a Taylor expansion of this nonlinear nonlocal term in $\varphi$, we get the desired nonlinear stability criterion.

Carrying out this approach in the RVM case is far from straightforward. One key point in our proof is to introduce the $\left|\mu_{e}\right|$-weighted $L^{2}$ space $L_{\left|\mu_{e}\right|}^{2}$ which contains both $f$ and $\psi$ and to perform the projection in this space. The Legendre-type transformation from $f$ to $\psi$ is also quite delicate and the spectral stability proof from [15] provides useful insights. We are able to get a nonlocal nonlinear term involving $\psi$ only. Performing a Taylor expansion of this new nonlinear term of $\psi$, we obtain the nonlinear stability criterion that the operator

$$
\tilde{\mathscr{L}}=-\partial_{x}^{2} h-2 \int \hat{v}_{2} \mu_{p}^{-} d v h-2 \int \hat{v}_{2} \mu_{e}^{-} \tilde{\mathscr{P}}^{-}\left[\hat{v}_{2} h\right] d v
$$

is nonnegative with $\operatorname{ker} \tilde{\mathscr{L}}=\operatorname{span}\left\{\partial_{x} \psi_{0}\right\}$, where $\tilde{\mathscr{P}}^{-}$is the projection operator of $L_{\mu_{-}}^{2}$ onto the subspace

(1.10) $W^{-}=\left\{\zeta \in L_{\mu^{-}}^{2} \mid \zeta=g\left(\langle v\rangle, v_{2}-\psi^{0}\right)\right.$ for some measurable function $\left.g\right\}$.

However, $W^{-}$is strictly smaller than $\operatorname{ker} D^{-}$, where $D^{-}$is the Vlasov generator, so that $\mathscr{P}^{-}>\tilde{\mathscr{P}}^{-}$. Therefore $\mathscr{L}^{0}>\tilde{\mathscr{L}}$. So as in the case of ideal plane flows there is a gap between the sharp linear stability criterion $\mathscr{L}^{0} \geq 0$ and the nonlinear stability criterion $\tilde{\mathscr{L}} \geq 0$. The reason for this gap is similar: the phase space for the particle motion for given particle invariants $e, p$ in a steady field has disconnected components. To close the gap between $\mathscr{L}^{0}$ and $\tilde{\mathscr{L}}$ and to get the sharp criterion for nonlinear stability, we investigate the detailed structure of particle trajectories. The 
key observation is that the disjoint components of the trapped intervals are symmetric in the purely magnetic case. Using this observation and the explicit forms of $\mathscr{L}^{0}$ and $\tilde{\mathscr{L}}$, we are able to show that $\mathscr{L}^{0} \geq 0$ implies $\tilde{\mathscr{L}} \geq 0$. This finally provides the sharp nonlinear stability result of Theorem 1.2. Using this sharp criterion, we can prove nonlinear stability under general perturbations for the stable examples constructed in [15].

This paper is organized as follows. In Section 2, we prove Theorem 1.2 on nonlinear stability. In Section 3, we prove Theorem 1.1 in several steps. First we discuss the averaging Liapunov exponent, then the representation of the fields, then the semigroup estimates, then the bootstrap estimate and finally the nonlinear stability.

\section{Sharp nonlinear stability in the purely magnetic case}

\subsection{The invariant functional and duality lemma}

We prove Theorem 1.2 in this section. Denote $H(f)=f \ln f-f$ and $\eta(p)=$ $\ln \gamma(p)$. Since $\int_{0}^{P} B(t)$ is invariant, $B(t)=\partial_{x} \psi(t)+k$ for a periodic $\psi(t)$ and a constant $k$ independent of $t$. To prove nonlinear stability, we only need to control the periodic part $\partial_{x} \psi(t)$. For simplicity, as in the previous papers [9] and [6], we only consider perturbations with $k=\int_{0}^{P} B(0)=0$. This allows us to write $B(t)=$ $\partial_{x} \psi(t)$, where $\psi(t)$ is the periodic magnetic potential. We define the standard energy-Casimir functional as in [6],

$$
I\left(f^{ \pm}, \psi, E\right)=
$$

$$
\int_{0}^{P} \int_{\mathbf{R}^{2}} \sum_{ \pm} H\left(f^{ \pm}\right)+\left(\langle v\rangle-\eta\left(v_{2} \pm \psi\right)\right) f^{ \pm} d v d x+\int_{0}^{P}\left(\frac{1}{2} \psi_{x}^{2}+\frac{1}{2}|E|^{2}\right) d x
$$

To prove nonlinear stability, we expand the functional (2.1) around the equilibrium. Denote

$$
Q\left(f^{ \pm}, v_{2} \pm \psi\right)=H\left(f^{ \pm}\right)-\eta\left(v_{2} \pm \psi\right) f^{ \pm}
$$

so that $\partial_{1} Q\left(\mu^{ \pm}, v_{2} \pm \psi^{0}\right)=-\langle v\rangle$. So from

$$
0=\int \partial_{v_{2}} Q\left(\mu^{ \pm}, v_{2} \pm \psi^{0}\right) d v=\int\left(-\langle v\rangle \partial_{v_{2}} \mu^{ \pm}-\eta^{\prime}\left(v_{2} \pm \psi^{0}\right) \mu^{ \pm}\right) d v
$$

we have

$$
\int \eta^{\prime}\left(v_{2} \pm \psi^{0}\right) \mu^{ \pm} d v=-\int\langle v\rangle \partial_{v_{2}} \mu^{ \pm} d v=\int \hat{v}_{2} \mu^{ \pm} d v
$$

and thus

$$
-\psi_{x x}^{0}=\int \hat{v}_{2}\left(\mu^{+}-\mu^{+}\right) d v=\int\left(\eta^{\prime}\left(v_{2}+\psi^{0}\right) \mu^{+}-\eta^{\prime}\left(v_{2}-\psi^{0}\right) \mu^{-}\right) d v
$$


NONLINEAR STABILITY AND INSTABILITY OF RUM

9

Hence

$$
\begin{aligned}
& I\left(f^{ \pm}, \psi, E\right)-I\left(\mu^{ \pm}, \psi^{0}, 0\right)=\iint \sum_{ \pm}\left[H\left(f^{ \pm}\right)-H\left(\mu^{ \pm}\right)\right] d v d x \\
& +\iint \sum_{ \pm}\left\{\langle v\rangle\left(f^{ \pm}-\mu^{ \pm}\right)-\eta\left(v_{2} \pm \psi\right) f^{ \pm}+\eta\left(v_{2} \pm \psi^{0}\right) \mu^{ \pm}\right\} d v d x \\
& +\int\left\{\frac{1}{2}\left(\psi-\psi^{0}\right)_{x}^{2}+\left(\psi-\psi^{0}\right)_{x} \psi_{0 x}+\frac{1}{2}|E|^{2}\right\} d x \\
& =\iint \sum_{ \pm}\left\{H\left(f^{ \pm}\right)-H\left(\mu^{ \pm}\right)+\langle v\rangle\left(f^{ \pm}-\mu^{ \pm}\right)-\eta\left(v_{2} \pm \psi\right) f^{ \pm}\right\} d v d x \\
& +\iint \sum_{ \pm}\left\{\eta\left(v_{2} \pm \psi^{0}\right) \mu^{ \pm}+\left(\eta^{\prime}\left(v_{2}+\psi^{0}\right) \mu^{+}-\eta^{\prime}\left(v_{2}-\psi^{0}\right) \mu^{-}\right)\left(\psi-\psi^{0}\right)\right\} d v d x \\
& +\int\left\{\frac{1}{2}\left(\psi-\psi^{0}\right)_{x}^{2}+\frac{1}{2}|E|^{2}\right\} d x \\
& =I_{1}+I_{2}+I_{3},
\end{aligned}
$$

where in the second equality above we have integrated by parts and used (2.2) and with

$$
I_{1}=\int_{0}^{P} \int_{\mathbb{R}^{2}}\left(\sum_{ \pm} H\left(f^{ \pm}\right)-H\left(\mu^{ \pm}\right)-H^{\prime}\left(\mu^{ \pm}\right)\left(f^{ \pm}-\mu^{ \pm}\right)-h\left(f^{ \pm}-\mu^{ \pm}\right)\right) d v d x
$$

where $h=\eta\left(v_{2} \pm \psi\right)-\eta\left(v_{2} \pm \psi^{0}\right)$,

$$
\begin{gathered}
I_{2}=-\int_{0}^{P} \int_{\mathbb{R}^{2}}\left(\sum_{ \pm}\left(h \mp \eta^{\prime}\left(v_{2} \pm \psi^{0}\right)\left(\psi-\psi^{0}\right)\right) \mu^{ \pm}\right) d v d x \\
I_{3}=+\int\left(\frac{1}{2}\left(\psi-\psi^{0}\right)_{x}^{2}+\frac{1}{2}|E|^{2}\right) d x
\end{gathered}
$$

Now we use the integral identity (which follows from the special form of $\mu$ )

$$
\begin{aligned}
\int \mu^{ \pm} \eta^{\prime \prime}\left(v_{2} \pm \psi^{0}\right) d v & =\int-\partial_{v_{2}}\left(\mu^{ \pm}\right) \eta^{\prime}\left(v_{2} \pm \psi^{0}\right) d v \\
& =\int\left(-\mu^{ \pm}\left(\eta^{\prime}\left(v_{2} \pm \psi^{0}\right)\right)^{2}+\hat{v}_{2} \mu^{ \pm} \eta^{\prime}\left(v_{2} \pm \psi^{0}\right)\right) d v \\
& =\int\left(-\mu^{ \pm}\left(\eta^{\prime}\left(v_{2} \pm \psi^{0}\right)\right)^{2}+\hat{v}_{2} \mu_{p}^{ \pm}\right) d v
\end{aligned}
$$


Thus the term $I_{2}$ is estimated below by

$$
\begin{aligned}
I_{2} & =-\int_{0}^{P} \int_{\mathbb{R}^{2}} \sum_{ \pm}\left(\frac{1}{2} \eta^{\prime \prime}\left(v_{2} \pm \psi^{0}\right)\left(\psi-\psi^{0}\right)^{2} \pm \eta^{\prime \prime \prime}\left(v_{2} \pm \bar{\psi}\right)\left(\psi-\psi^{0}\right)^{3}\right) \mu^{ \pm} d x d v \\
& \geq \int_{0}^{P} \int_{\mathbb{R}^{2}} \sum_{ \pm}-\frac{1}{2} \mu^{ \pm} \eta^{\prime \prime}\left(v_{2} \pm \psi^{0}\right)\left(\psi-\psi^{0}\right)^{2} d x d v-C_{1}\left\|\psi-\psi^{0}\right\|_{H^{1}}^{3} \\
& =\int_{0}^{P} \int_{\mathbb{R}^{2}} \sum_{ \pm}\left(\frac{1}{2} \mu^{ \pm}\left(\eta^{\prime}\left(v_{2} \pm \psi^{0}\right)\right)^{2}-\frac{1}{2} \hat{v}_{2} \mu_{p}^{ \pm}\right)\left(\psi-\psi^{0}\right)^{2} d v d x-C_{1}\left\|\psi-\psi^{0}\right\|_{H^{1}}^{3}
\end{aligned}
$$

where $C_{1}=\left|\eta^{\prime \prime \prime}\right|_{\mathbf{L}^{\infty}} \int \sum_{ \pm}\left|\mu^{ \pm}\right| d x d v$ and $\bar{\psi}$ is between $\psi^{0}$ and $\psi$. In order to estimate $I_{1}$, we need the following simple duality formula based on the Legendre transform.

Lemma 2.1. For given numbers $f_{0}>0, c$ and d, denote $g_{c, d}(f)=H\left(f+f_{0}+d\right)-$ $H\left(f_{0}\right)-H^{\prime}\left(f_{0}\right)(f+d)-c f$. Then

$$
\begin{aligned}
G_{c, d}(h) & =\min _{f>-\left(f_{0}+d\right)} g_{c, d}(f)-h f \\
& =f_{0}\left(1+h+c-e^{h+c}\right)+(h+c) d=(h+c) d+O(h+c)^{2} .
\end{aligned}
$$

Proof. The minimizer $f_{h}$ satisfies

$$
0=g_{c, d}^{\prime}(f)-h=\ln \left(f+f_{0}+d\right)-\ln f_{0}-h-c=0,
$$

so

$$
f_{h}=f_{0}\left(e^{h+c}-1\right)-d
$$

Thus

$$
G_{c, d}(h)=g_{c, d}\left(f_{h}\right)-h f_{h}=f_{0}\left(1+h+c-e^{h+c}\right)+(h+c) d .
$$

As a simple illustration of estimating $I_{1}$ by the duality argument, we use Lemma 2.1 by setting $c=d=0, f_{0}=\mu^{ \pm}, f=f^{ \pm}-\mu^{ \pm}$and $h=h^{ \pm}\left(\psi, \psi^{0}\right)=\eta\left(v_{2} \pm \psi\right)-$ $\eta\left(v_{2} \pm \psi^{0}\right)$. Then we have by Lemma 2.1,

$$
\begin{aligned}
I_{1}= & \int_{0}^{P} \int_{\mathbb{R}^{2}} \sum_{ \pm}\left(g_{0,0}\left(f^{ \pm}\right)-h\right)\left(f^{ \pm}-\mu^{ \pm}\right) d v d x \\
\geq & \int_{0}^{P} \int_{\mathbb{R}^{2}} \sum_{ \pm} \mu^{ \pm}\left(1+h^{ \pm}\left(\psi, \psi^{0}\right)-e^{h^{ \pm}\left(\psi, \psi_{0}\right)}\right) d v d x \\
\geq & -\int_{0}^{P} \int_{\mathbb{R}^{2}} \sum_{ \pm} \frac{1}{2} \mu^{ \pm}\left(\eta^{\prime}\left(v_{2} \pm \psi^{0}\right)\right)^{2}\left(\left(\psi-\psi^{0}\right)^{2}\right) d v d x \\
& -C_{1} e^{C^{\prime}}\left\|\psi-\psi^{0}\right\|_{H^{1}}\left\|\psi-\psi^{0}\right\|_{H^{1}}^{3}
\end{aligned}
$$


since $h^{ \pm}\left(\psi, \psi^{0}\right) \leq\left|\eta^{\prime}\right|_{L^{\infty}}\left|\psi-\psi^{0}\right| \leq C^{\prime}\left\|\psi-\psi^{0}\right\|_{H^{1}}$. Combining this with the estimate (2.4) of $I_{2}$, we get a cancelation that leads to

$$
\begin{aligned}
I\left(f^{ \pm}, \psi, E\right)-I\left(\mu^{ \pm}, \psi^{0}, 0\right) & \geq \frac{1}{2}\|E\|_{2}^{2}+\left(L\left(\psi-\psi_{0}\right), \psi-\psi^{0}\right) \\
& -C_{1} e^{C^{\prime}}\left\|\psi-\psi^{0}\right\|_{H^{1}}\left\|\psi-\psi^{0}\right\|_{H^{1}}^{3} .
\end{aligned}
$$

By the standard argument, this essentially proves nonlinear stability under the stronger assumption that the operator $L$ defined by (1.8) is positive, which recovers the result of Guo [6].

However, the proof of the nonlinear stability criterion asserted in Theorem 1.2 is sharper and its proof is considerably more involved. We divide it into several steps. Our main task is to use the constrained energy-Casimir method to show that $\tilde{\mathscr{L}} \geq 0$ (defined by (1.9)) implies nonlinear stability in the sense of Theorem 1.2. Assuming that, we will finish the proof of Theorem 1.2 by showing that $\tilde{\mathscr{L}} \geq 0$ is equivalent to the sharp condition $\mathscr{L}^{0} \geq 0$.

\subsection{Two projections}

We begin with a discussion of the operator $\tilde{\mathscr{L}}$ defined by (1.9). Since we do not know beforehand that the space $W^{-}$(defined by (1.10)) is closed in $L_{\left|\mu_{e}^{-}\right|}^{2}$, we shall define an operator $\tilde{\mathscr{P}}^{-}: L_{\left|\mu_{e}^{-}\right|}^{2} \rightarrow L_{\left|\mu_{e}^{-}\right|}^{2}$ explicitly and later show that it is the projection onto $W^{-}$. For $h=h\left(x, v_{1}, v_{2}\right) \in L_{\left|\mu_{e}^{-}\right|}^{2}$, we denote

$$
\tilde{h}^{ \pm}(e, p, x)=h\left(x, \pm \sqrt{e^{2}-1-\left(p+\psi^{0}(x)\right)^{2}}, p+\psi^{0}(x)\right) .
$$

We classify the particles into five types according to their $(e, p)$ values as in [15] and we refer to [15] for their definitions and the notation. The classification depends on which of five sets $A_{1} \cup \cdots \cup A_{5}$ the point $(e, p)$ belongs to. We can neglect the set $A_{5}$, which has measure zero. We define

$$
\tilde{\mathscr{P}}^{-} h=\frac{1}{4 T(e, p)} \sum_{i=1,2} \sum_{ \pm} \int_{a_{i}}^{b_{i}} \frac{\tilde{h}^{ \pm}(e, p, x) e d x}{\sqrt{e^{2}-1-\left(p+\psi^{0}(x)\right)^{2}}}
$$

at points $(x, v)$ with $(e, p)=\left(\langle v\rangle, v_{2}-\psi^{0}(x)\right) \in A_{4}$ corresponding to trapped particles of type III, where $\left[a_{1}^{I I I}, b_{1}^{I I I}\right]$ and $\left[a_{2}^{I I I}, b_{2}^{I I I}\right]$ are trapped intervals and

$$
T^{I I I}(e, p)=\int_{a_{i}^{I I I}}^{b_{i}^{I I I}} \frac{e d x}{\sqrt{e^{2}-1-\left(p+\psi^{0}(x)\right)^{2}}}
$$

is the half period. At all other points $(e, p) \in A_{1} \cup A_{2} \cup A_{3}$, we define

$$
\tilde{\mathscr{P}}^{-} h=\frac{1}{2 T(e, p)} \sum_{ \pm} \int_{a}^{b} \frac{\tilde{h}^{ \pm}(e, p, x) e d x}{\sqrt{e^{2}-1-\left(p+\psi^{0}(x)\right)^{2}}}
$$


where $[a, b]$ is the interval of the particle motion and

$$
T(e, p)=\int_{a}^{b} \frac{e d x}{\sqrt{e^{2}-1-\left(p+\psi^{0}(x)\right)^{2}}}
$$

is the time for a particle to get through its interval. By definition, $\tilde{\mathscr{P}}^{-}$takes an arbitrary function into a function of $e, p$ alone. We now show that $\tilde{\mathscr{P}}^{-}$, as just defined, is the projection onto $W^{-}$.

Lemma 2.2. (i) $\tilde{\mathscr{P}}^{-}: L_{\left|\mu_{e}^{-}\right|}^{2} \rightarrow L_{\left|\mu_{e}^{-}\right|}^{2}$ is the orthogonal projection onto $W^{-}$.

(ii) If $\psi(x) \in L_{P}^{2}$ is odd, then $\tilde{\mathscr{P}}^{-}\left(\hat{v}_{2} \psi\right)=0$. If $\psi$ is even, then $\tilde{\mathscr{P}}^{-}\left(\hat{v}_{2} \psi\right)=$ $\mathscr{P}^{-}\left(\hat{v}_{2} \psi\right)$ is even.

Proof. (i) We have

$$
\begin{aligned}
\|h\|_{L_{\left|\mu_{e}^{-}\right|}^{2}}^{2} & =\int\left|\mu_{e}^{-}\right| h^{2} d x d v \\
& =\iint_{A_{1} \cup A_{2} \cup A_{3}}\left|\mu_{e}^{-}\right| \sum_{ \pm} \int_{a}^{b} \frac{\tilde{h}^{ \pm}(e, p, x)^{2} e d x}{\sqrt{e^{2}-1-\left(p+\psi^{0}(x)\right)^{2}}} d e d p \\
& +\iint_{A_{4}}\left|\mu_{e}^{-}\right| \sum_{ \pm} \sum_{i=1,2} \int_{a_{i}}^{b_{i}} \frac{\tilde{h}^{ \pm}(e, p, x)^{2} e d x}{\sqrt{e^{2}-1-\left(p+\psi^{0}(x)\right)^{2}}} d e d p
\end{aligned}
$$

by the change of variables $\left(x, v_{1}, v_{2}\right) \rightarrow(x, e, p)$. Since by definition $\tilde{\mathscr{P}}^{-} h$ depends only on $(e, p)$, it is constant on the $x$-intervals for each $(e, p)$, so that

$$
\begin{aligned}
& \left\|\tilde{\mathscr{P}}^{-} h\right\|_{L_{\left|\mu_{e}^{-}\right|}^{2}}^{2}=\iiint\left|\mu_{e}^{-}\right|\left(\tilde{\mathscr{P}}^{-} h\right)^{2} d x d v \\
& =2 \iint_{A_{1} \cup A_{2} \cup A_{3}}\left|\mu_{e}^{-}\right|\left(\tilde{\mathscr{P}}^{-} h\right)^{2} T(e, p) \operatorname{ded} p+4 \iint_{A_{4}}\left|\mu_{e}^{-}\right|\left(\tilde{\mathscr{P}}^{-} h\right)^{2} T^{I I I}(e, p) \operatorname{ded} p .
\end{aligned}
$$

By Cauchy-Schwarz, for $(e, p) \in A_{1} \cup A_{2} \cup A_{3}$,

$$
\begin{aligned}
& \left(\tilde{\mathscr{P}}^{-} h\right)^{2} 2 T(e, p) \\
& \leq \frac{1}{2 T(e, p)} \sum_{ \pm}\left(\int_{a}^{b} \frac{\tilde{h}^{ \pm}(e, p, x)^{2} e d x}{\sqrt{e^{2}-1-\left(p+\psi^{0}(x)\right)^{2}}}\right)\left(\int_{a}^{b} \frac{e d x}{\sqrt{e^{2}-1-\left(p+\psi^{0}(x)\right)^{2}}}\right)^{\frac{1}{2}} \\
& =\frac{1}{2} \sum_{ \pm} \int_{a}^{b} \frac{\tilde{h}^{ \pm}(e, p, x)^{2} e d x}{\sqrt{e^{2}-1-\left(p+\psi^{0}(x)\right)^{2}}} \leq \sum_{ \pm} \int_{a}^{b} \frac{\tilde{h}^{ \pm}(e, p, x)^{2} e d x}{\sqrt{e^{2}-1-\left(p+\psi^{0}(x)\right)^{2}}} .
\end{aligned}
$$


Similarly for $(e, p) \in A_{4}$,

$$
\begin{aligned}
4\left(\tilde{\mathscr{P}}^{-} h\right)^{2} T^{I I I}(e, p) & \leq \frac{1}{4}\left(\sum_{ \pm} \sum_{i=1,2}\left(\int_{a_{i}^{I I I}}^{b_{i}^{I I}} \frac{\tilde{h}^{ \pm}(e, p, x)^{2} e d x}{\sqrt{e^{2}-1-\left(p+\psi^{0}(x)\right)^{2}}}\right)^{\frac{1}{2}}\right)^{2} \\
& \leq \sum_{ \pm} \sum_{i=1,2} \int_{a_{i}^{I I I}}^{b_{i}^{I I I}} \frac{\tilde{h}^{ \pm}(e, p, x)^{2} e d x}{\sqrt{e^{2}-1-\left(p+\psi^{0}(x)\right)^{2}}} .
\end{aligned}
$$

So, summing over the four regions, we have $\left\|\tilde{\mathscr{P}}^{-} h\right\|_{L_{\left|\mu_{e}^{2}\right|}^{2}} \leq\|h\|_{L_{\left|\mu_{e}^{-}\right|}^{2}}$ or $\left\|\tilde{\mathscr{P}}^{-}\right\| \leq$ 1. That range $\left(\tilde{\mathscr{P}}^{-}\right)=W^{-}$is obvious since $\tilde{\mathscr{P}}^{-}$maps a function depending only on $e, p$ to the same kind of function. The equality $\left(\tilde{\mathscr{P}}^{-}\right)^{2}=\tilde{\mathscr{P}}^{-}=\left(\tilde{\mathscr{P}}^{-}\right)^{*}$ follows directly from the definition.

(ii) By the definition of $\tilde{\mathscr{P}}^{-}$, we have

$$
\tilde{\mathscr{P}}^{-}\left(\hat{v}_{2} \psi\right)=\frac{1}{2 T(e, p)} \sum_{i=1,2} \int_{a_{i}^{I I I}}^{b_{i}^{I I I}} \frac{\left(p+\psi^{0}(x)\right) \psi(x) d x}{\sqrt{e^{2}-1-\left(p+\psi^{0}(x)\right)^{2}}}
$$

for $(x, v)$ corresponding to type III trapped particles, and

$$
\tilde{\mathscr{P}}^{-}\left(\hat{v}_{2} \psi\right)=\frac{1}{T(e, p)} \int_{a}^{b} \frac{\left(p+\psi^{0}(x)\right) \psi(x) d x}{\sqrt{e^{2}-1-\left(p+\psi^{0}(x)\right)^{2}}}
$$

at all other points. So (ii) follows easily from this formula for $\tilde{\mathscr{P}}^{-}\left(\hat{v}_{2} \psi\right)$ and the formula for $\mathscr{P}^{-}\left(\hat{v}_{2} \psi\right)$ in [15], by the exact cancellation due to the symmetry properties of the particle motion intervals.

By the same process as above, we define $\tilde{\mathscr{P}}^{+}$to be the projection operator of $L_{\left|\mu_{e}^{+}\right|}^{2}$ to its subspace $W^{+}=\left\{\zeta \in L_{\left|\mu_{e}^{+}\right|}^{2} \mid \zeta=g\left(\langle v\rangle, v_{2}+\psi^{0}\right)\right\}$. Let $R$ be the operator defined by $R f(x, v)=f(x,-v)$. Note that $R L_{\mu^{-}}^{2}=L_{\mu^{+}}^{2}$ and $R W^{-}=W^{+}$. Thus $R \mathscr{P}^{-}=\mathscr{P}^{+} R$.

The following lemma implies that the weaker stability criterion $\tilde{\mathscr{L}} \geq 0$ is equivalent to the sharp condition $\mathscr{L}^{0} \geq 0$. This is a crucial step in the proof of the sharp nonlinear stability criterion.

Lemma 2.3. $\mathscr{L}^{0} \geq 0$ with $\operatorname{ker} \mathscr{L}^{0}=\operatorname{span}\left\{\psi_{0 x}\right\}$ if and only if $\tilde{\mathscr{L}} \geq 0$ with $\operatorname{ker} \tilde{\mathscr{L}}=$ $\operatorname{span}\left\{\psi_{0 x}\right\}$.

Proof. Since $\tilde{\mathscr{L}}$ and $\mathscr{L}$ map odd (even) functions to odd (even) functions, we can consider the operators $\tilde{\mathscr{L}}$ and $\mathscr{L}$ defined on even and odd function spaces separately. By Lemma $2.2, \mathscr{L}=\tilde{\mathscr{L}}$ on the space of even functions. Since $\psi_{0 x}$ is odd, by assumption $\tilde{\mathscr{L}}=\mathscr{L}>0$ on the even space. On the odd space, $\tilde{\mathscr{L}}=L$ 
(defined by (1.8)) which is nonnegative by assumption and has its kernel spanned by $\psi_{0 x}$ on the odd space. Combining these two, we conclude the proof.

\subsection{Reduction to finitely many constraints}

By Lemma 2.2 it suffices to prove nonlinear stability under the assumption that $\tilde{\mathscr{L}} \geq 0$ and $\operatorname{ker} \tilde{\mathscr{L}}=\operatorname{span}\left\{\psi_{0 x}\right\}$. To accomplish this, the main idea is to use all the constraints from the invariance of $g\left(f^{ \pm}, v_{2} \pm \psi\right)$ to obtain a sharper estimate of $I\left(f^{ \pm}, \psi, E\right)-I\left(\mu^{ \pm}, \psi^{0}, 0\right)$. First we indicate that in some sense we merely need to use finitely many constraints. Indeed, for any $\zeta=g\left(\langle v\rangle, v_{2}-\psi^{0}\right)=g(e, p) \in W^{-}$, we denote $e^{\prime}=e-\eta(p)$ where $\eta=\log \gamma$. Then $\zeta=g\left(e^{\prime}+\eta(p), p\right)=\tilde{g}\left(e^{\prime}, p\right)$.

So we also have $W^{-}=\left\{\zeta \in L_{\left|\mu_{e}^{-}\right|}^{2} \mid \zeta=\tilde{g}\left(e^{\prime}, p\right)\right\}$, and

$$
\|\zeta\|_{L_{\left|\mu_{e}^{2}\right|}^{2}}^{2}=\int\left|\mu_{e}^{-}\right| \zeta^{2} d x d v=\iint\left|\mu_{e}^{-}\right| g^{2}\left(e^{\prime}, p\right) \tilde{T}\left(e^{\prime}, p\right) d e^{\prime} d p
$$

where $\tilde{T}\left(e^{\prime}, p\right)=T\left(e^{\prime}+\eta(p), p\right)$ and

$$
T(e, p)=\int_{a}^{b} \frac{e d x}{\sqrt{e^{2}-1-\left(p+\psi^{0}(x)\right)^{2}}}
$$

is the time for a particle to get through its periodic interval $[a, b]$. Hence the space $\left(W^{-},\|\cdot\|_{L_{\left|\mu_{e}^{-}\right|}^{2}}\right)$ is the same as the $\left|\mu_{e}^{-}\right| \tilde{T}$ weighted $L_{e^{\prime}, p}^{2}$ space. Therefore we can find a complete orthogonal basis $\left\{\zeta_{1}, \zeta_{2}, \cdots, \zeta_{n}, \cdots\right\}$ for $\left(W^{-},\|\cdot\|_{L_{\left|\mu_{e}^{-}\right|}^{2}}\right)$ in the form of products

$$
\zeta_{i}=\alpha_{i}\left(e^{\prime}\right) \beta_{i}(p)=\alpha_{i}\left(\langle v\rangle-\eta\left(v_{2}-\psi^{0}(x)\right)\right) \beta_{i}\left(v_{2}-\psi^{0}(x)\right)
$$

with $\alpha_{i}, \beta_{i}$ in $C_{0}^{2}(\mathbb{R})$. Denote $W_{m}^{-}=\operatorname{span}\left\{\zeta_{1}, \zeta_{2}, \cdots, \zeta_{m}\right\}$ and $\tilde{\mathscr{P}}_{m}^{-}$the corresponding projection operator from $L_{\left|\mu_{e}^{-}\right|}^{2}$ to $W_{m}^{-}$. Similarly, for any $m$, denote $W_{m}^{+}$ $=\operatorname{span}\left\{\zeta_{1}^{+}, \zeta_{2}^{+}, \cdots, \zeta_{m}^{+}\right\} \subset L_{\left|\mu_{e}^{+}\right|}^{2}$ with

$$
\zeta_{i}^{+}=\alpha_{i}\left(\langle v\rangle-\eta\left(v_{2}+\psi^{0}\right)\right) \beta_{i}\left(v_{2}+\psi^{0}\right)
$$

and $\tilde{\mathscr{P}}_{m}^{+}$the corresponding projection operator from $L_{\left|\mu_{e}^{+}\right|}^{2}$ to $W_{m}^{+}$. Define

$$
\tilde{\mathscr{L}}_{m} h=-\partial_{x}^{2} h-\sum_{ \pm} \int \hat{v}_{2} \mu_{p}^{ \pm} d v h-\sum_{ \pm} \int \hat{v}_{2} \mu_{e}^{ \pm} \tilde{\mathscr{P}}_{m}^{ \pm}\left[\hat{v}_{2} h\right] d v .
$$

Lemma 2.4. Assume $\tilde{\mathscr{L}} \geq 0$ and $\operatorname{ker} \tilde{\mathscr{L}}=\left\{\psi_{x}^{0}\right\}$. For sufficiently large $m$, the operator $\tilde{\mathscr{L}}_{m}$ defined by (2.6) satisfies $\tilde{\mathscr{L}}_{m} \geq 0$ and $\operatorname{ker} \tilde{\mathscr{L}}_{m}=\left\{\psi_{x}^{0}\right\}$. 
Proof. Since $\hat{v}_{2} \psi_{x}^{0}=D^{-}\left(v_{1}\right)$, we have $\hat{v}_{2} \psi_{x}^{0} \in\left(W_{m}^{-}\right)^{\perp}$ and $\tilde{\mathscr{P}}_{m}^{-}\left[\hat{v}_{2} \psi_{x}^{0}\right]=0$. Similarly, $\tilde{\mathscr{P}}_{m}^{+}\left[\hat{v}_{2} \psi_{0 x}\right]=0$. So $\tilde{\mathscr{L}}_{m}\left(\psi_{0 x}\right)=L \psi_{0 x}=0$. It is obvious that $\tilde{\mathscr{P}}_{m}^{ \pm} \rightarrow \tilde{\mathscr{P}}^{ \pm}$ strongly in $L_{\left|\mu_{e}^{-}\right|}^{2}$, so

$$
\begin{aligned}
\left\|\tilde{\mathscr{L}}_{m} h-\tilde{\mathscr{L}} h\right\|_{2} & \leq \sum_{ \pm}\left(\int\left|\int \hat{v}_{2} \mu_{e}^{ \pm}\left(\tilde{\mathscr{P}}_{m}^{ \pm}\left[\hat{v}_{2} h\right]-\tilde{\mathscr{P}}^{ \pm}\left[\hat{v}_{2} h\right]\right) d v\right|^{2} d x\right)^{\frac{1}{2}} \\
& \leq C \sum_{ \pm}\left(\iint\left|\mu_{e}^{ \pm}\right|\left|\tilde{\mathscr{P}}_{m}^{ \pm}\left[\hat{v}_{2} h\right]-\tilde{\mathscr{P}}^{ \pm}\left[\hat{v}_{2} h\right]\right|^{2} d v d x\right)^{\frac{1}{2}} \rightarrow 0
\end{aligned}
$$

as $m \rightarrow \infty$. Thus $\tilde{\mathscr{L}}_{m} \rightarrow \tilde{\mathscr{L}}$ strongly in $L_{\text {per }}^{2}$, as $m \rightarrow \infty$. We are assuming $\tilde{\mathscr{L}}>0$ on $\left\{\psi_{x}^{0}\right\}^{\perp}$ and we know its spectrum is discrete.

Suppose that $\tilde{\mathscr{L}}_{m} \ngtr 0$ on $\left\{\psi_{x}^{0}\right\}^{\perp}$ for some large $m$. Then there exists a sequence $\left\{\lambda_{n}\right\}$ and $\left\{\phi_{n}\right\} \subset\left\{\psi_{x}^{0}\right\}^{\perp}$ with $\lambda_{n}<0,\left\|\phi_{n}\right\|_{2}=1$ and $\tilde{\mathscr{L}}_{n} \phi_{n}=\lambda_{n} \phi_{n}$. Since the operators $\mathscr{L}_{m}+\partial_{x}^{2}$ are uniformly bounded in $L_{p e r}^{2}$, standard elliptic estimates imply that $\left\|\phi_{n}\right\|_{H_{p e r}^{2}} \leq C$ for some constant independent of $n$. So there exists $\lambda_{0} \leq 0$ and $\phi_{0} \in H_{p e r}^{2}$ such that $\lambda_{n} \rightarrow \lambda_{0}$ and $\phi_{n} \rightarrow \phi_{0}$ strongly in $L_{p e r}^{2}$. So $\tilde{\mathscr{L}}_{n} \phi_{n} \rightarrow \tilde{\mathscr{L}} \phi_{0}$ weakly and thus $\tilde{\mathscr{L}} \phi_{0}=\lambda_{0} \phi_{0}$, a contradiction. Thus $\mathscr{L}_{m}>0$ on $\left\{\psi_{0 x}\right\}^{\perp}$.

\subsection{Distance functionals}

By assumption, $\mathscr{L}^{0} \geq 0$ and $\operatorname{ker} \mathscr{L}^{0}=\operatorname{span}\left\{\psi_{x}^{0}\right\}$. So from Lemmas 2.3 and 2.4 , there exists a $m$-dimensional subspace $W_{m}^{-}\left(W_{m}^{-}\right)$in $L_{\left|\mu_{e}^{-}\right|}^{2}\left(L_{\left|\mu_{e}^{+}\right|}^{2}\right)$ with projection $\tilde{\mathscr{P}}_{m}^{-}\left(\tilde{\mathscr{P}}_{m}^{+}\right)$as defined above such that $\tilde{\mathscr{L}}_{m} \geq 0$ and $\operatorname{ker} \tilde{\mathscr{L}}_{m}=\left\{\psi_{x}^{0}\right\}$. To quantify the nonlinear stability, we introduce the following distance functional

$$
d\left(f^{ \pm}-\mu^{ \pm}, \psi-\psi^{0}, E\right)=\left\|\psi-\psi^{0}\right\|_{H^{1}}^{2}+\|E\|_{2}^{2}+\sum_{ \pm} d_{1}^{ \pm}\left(f^{ \pm}, \mu^{ \pm}\right)
$$

where

$$
d_{H}^{ \pm}=d_{1}^{ \pm}\left(f^{ \pm}, \mu^{ \pm}\right)=\int_{0}^{P} \int_{\mathbb{R}^{2}}\left(H\left(f^{ \pm}\right)-H\left(\mu^{ \pm}\right)-H^{\prime}\left(\mu^{ \pm}\right)\left(f^{ \pm}-\mu^{ \pm}\right)\right) d v d x .
$$

We note that by Taylor's formula

$$
d_{1}^{ \pm}\left(f^{ \pm}, \mu^{ \pm}\right)=\int_{0}^{1} \int_{0}^{P} \int_{\mathbb{R}^{2}}(1-r) \frac{1}{f^{ \pm, r}}\left(f^{ \pm}-\mu^{ \pm}\right)^{2} d v d x d r \geq 0
$$


where $f^{ \pm, r}=r f^{ \pm}+(1-r) \mu^{ \pm}$. So by the Cauchy-Schwarz inequality

$$
\begin{aligned}
\left\|f^{ \pm}-\mu^{ \pm}\right\|_{1} & =2 \int_{0}^{1} \int_{0}^{P} \int_{\mathbb{R}^{2}}(1-r)\left|f^{ \pm}-\mu^{ \pm}\right| d v d x d r \\
& \leq \int_{0}^{1} 2(1-r)\left(\int_{0}^{P} \int_{\mathbb{R}^{2}} \frac{1}{f^{ \pm, r}}\left(f^{ \pm}-\mu^{ \pm}\right)^{2} d v d x\right)^{\frac{1}{2}}\left(\int_{0}^{P} \int_{\mathbb{R}^{2}} f^{ \pm, r} d v d x\right)^{\frac{1}{2}} d r \\
& \leq C\left(\left\|f^{ \pm}(0)\right\|_{1},\left\|\mu^{ \pm}\right\|\right) d_{1}^{ \pm}\left(f^{ \pm}, \mu^{ \pm}\right)^{\frac{1}{2}} .
\end{aligned}
$$

\subsection{Projection based on the constraints}

Recall that $W_{m}^{-}=\operatorname{span}\left\{\zeta_{1}, \zeta_{2}, \cdots, \zeta_{m}\right\}$ with $\zeta_{i}=\alpha_{i}\left(\langle v\rangle-\eta\left(v_{2}-\psi^{0}\right)\right) \beta_{i}\left(v_{2}-\psi^{0}\right)$ for some functions $\alpha_{i}, \beta_{i} \in \mathbf{C}_{0}^{2}(\mathbb{R})$. Define

$$
A_{i}(f)=\int_{0}^{f} \alpha_{i}(-\ln s) d s, \quad Q_{i}\left(f, v_{2}-\psi\right)=A_{i}(f) \beta_{i}\left(v_{2}-\psi\right) .
$$

Note that $\partial_{1} Q_{i}\left(\mu^{-}, v_{2}-\psi^{0}\right)=\alpha_{i}\left(-\ln \mu^{-}\right) \beta_{i}\left(v_{2}-\psi^{0}\right)=\zeta_{i}$. We define the $m$ invariant functionals

$$
J_{i}\left(f^{-}, \psi\right)=\int_{0}^{P} \int_{\mathbb{R}^{2}} Q_{i}\left(f^{-}, v_{2}-\psi\right) d v d x
$$

In the following we investigate what estimates on perturbations $\left(f^{-}-\mu^{-}, \psi-\psi^{0}\right)$ can be derived from these additional invariants.

We perform a Taylor expansion of

$$
\begin{aligned}
& J_{i}\left(f^{-}, \psi\right)-J_{i}\left(\mu^{-}, \psi^{0}\right) \\
& =\int_{0}^{P} \int_{\mathbb{R}^{2}}\left(\partial_{1} Q_{i}\left(\mu^{-}, v_{2}-\psi^{0}\right)\left(f^{-}-\mu^{-}\right)-\partial_{2} Q_{i}\left(\mu^{-}, v_{2}-\psi^{0}\right)\left(\psi-\psi^{0}\right)\right) d v d x \\
& \quad+\int_{0}^{1}(1-r) \int_{0}^{P} \int_{\mathbb{R}^{2}} \partial_{11} Q_{i}\left(f^{-, r}, v_{2}-\psi^{r}\right)\left(f^{-}-\mu^{-}\right)^{2} d v d x d r \\
& \quad-\int_{0}^{1}(1-r) \int_{0}^{P} \int_{\mathbb{R}^{2}} \partial_{12} Q_{i}\left(f^{-, r}, v_{2}-\psi^{r}\right)\left(f^{-}-\mu^{-}\right)\left(\psi-\psi^{0}\right) d v d x d r \\
& \quad+\int_{0}^{1}(1-r) \int_{0}^{P} \int_{\mathbb{R}^{2}} \partial_{22} Q_{i}\left(f^{-, r}, v_{2}-\psi^{r}\right)\left(\psi-\psi^{0}\right)^{2} d v d x d r \\
& =K_{i}^{1}+\cdots K_{i}^{4},
\end{aligned}
$$

where for brevity we denote $f^{-, r}=r f^{-}+(1-r) \mu^{-}$and $\psi^{r}=r \psi+(1-r) \psi^{0}$. Since $\partial_{1} Q_{i}\left(\mu^{-}, v_{2}-\psi^{0}\right)=\zeta_{i}$ and

$$
\begin{aligned}
\int \partial_{2} Q_{i}\left(\mu^{-}, v_{2}-\psi^{0}\right) d v & =\int \partial_{v_{2}} Q_{i}\left(\mu^{-}, v_{2}-\psi^{0}\right) d v-\int \partial_{v_{2}} \mu^{-} \partial_{1} Q_{i}\left(\mu^{-}, v_{2}-\psi^{0}\right) d v \\
& =-\int \partial_{v_{2}} \mu^{-} \zeta_{i} d v
\end{aligned}
$$


the first term is

$$
K_{i}^{1}=\int_{0}^{P} \int_{\mathbb{R}^{2}}\left[\left(f^{-}-\mu^{-}\right)+\partial_{v_{2}} \mu^{-}\left(\psi-\psi^{0}\right)\right] \zeta_{i} d v d x .
$$

The second term can be estimated as

$$
\begin{aligned}
\left|K_{i}^{2}\right| & =\left|\int_{0}^{1}(1-r) \int_{0}^{P} \int_{\mathbb{R}^{2}} \alpha_{i}^{\prime}\left(-\ln f^{-, r}\right) \frac{1}{f^{-, r}}\left(f^{-}-\mu^{-}\right)^{2} d v d x d r\right| \\
& \leq\left|\alpha_{i}^{\prime}\right|_{\infty} \int_{0}^{1}(1-r) \int_{0}^{P} \int_{\mathbb{R}^{2}} \frac{1}{f^{-, r}}\left(f^{-}-\mu^{-}\right)^{2} d v d x d r=\left|\alpha_{i}^{\prime}\right|_{\infty} d_{1}^{-}\left(f^{-}, \mu^{-}\right) .
\end{aligned}
$$

For the third term we have

$$
\begin{aligned}
\left|K_{i}^{3}\right| & \leq\left|\alpha_{i}\right|_{\infty}\left|\beta_{i}^{\prime}\right|_{\infty}\left|\psi-\psi^{0}\right|_{\infty}\left\|f^{ \pm}-\mu^{ \pm}\right\|_{1} \\
& \leq C\left(\left\|f^{ \pm}(0)\right\|_{1},\left\|\mu^{ \pm}\right\|\right)\left\|\psi-\psi^{0}\right\|_{H^{1}} d_{1}^{-}\left(f^{-}, \mu^{-}\right)^{\frac{1}{2}}
\end{aligned}
$$

by (2.8). For the last term, noticing that $\left|A_{i}(f)\right| \leq\left|\alpha_{i}\right|_{\infty} f$, we have

$$
\begin{aligned}
\left|K_{i}^{4}\right| & \leq\left|\alpha_{i}\right|_{\infty}\left|\beta^{\prime \prime}\right|_{\infty}\left|\psi-\psi^{0}\right|_{\infty}^{2} \int_{0}^{1}\left\|f^{-, r}\right\|_{1} d r \\
& \leq C\left(\left\|f^{ \pm}(0)\right\|_{1},\left\|\mu^{ \pm}\right\|\right)\left\|\psi-\psi^{0}\right\|_{H^{1}}^{2}
\end{aligned}
$$

for some constant $C$. Now we use the estimate

$$
\left|J_{i}\left(f^{-}(0), \psi\right)-J_{i}\left(\mu^{-}(0), \psi^{0}\right)\right| \leq C\left(\left\|f^{ \pm}(0)\right\|_{1},\left\|\mu^{ \pm}\right\|\right)\left(d(0)^{\frac{1}{2}}+d(0)\right),
$$

which follows from

$$
\left|K_{i}^{1}\right|(0) \leq C\left(\left\|f^{-}(0)-\mu^{-}\right\|_{1}+\left\|\psi-\psi^{0}\right\|_{\infty}\right) \leq C d(0)^{\frac{1}{2}}
$$

by (2.8) and the existing estimates on terms $K_{i}^{2}(0), K_{i}^{3}(0), K_{i}^{4}(0)$. By the invariance of the functional $J_{i}\left(f^{-}(t), \psi(t)\right)$, the above calculations imply that at every time we have

$$
\begin{aligned}
& \left|\int_{0}^{P} \int_{\mathbb{R}^{2}} \zeta_{i}\left(\left(f^{-}(t)-\mu^{-}\right)+\partial_{v_{2}} \mu^{-}\left(\psi(t)-\psi^{0}\right)\right) d v d x\right| \\
& \leq\left|J_{i}\left(f^{-}(0), \psi\right)-J_{i}\left(\mu^{-}(0), \psi^{0}\right)\right|+C\left(\left\|f^{ \pm}(0)\right\|\left\|_{1},\right\| \mu^{ \pm} \|\right) d(t) \\
& \leq C\left(\left\|f^{ \pm}(0)\right\|_{1},\left\|\mu^{ \pm}\right\|\right)\left(d(0)^{\frac{1}{2}}+d(0)+d(t)\right),
\end{aligned}
$$

where $d(t)$ is the distance functional $d(t)=d\left(f^{ \pm}(t)-\mu^{ \pm}(t), \psi(t)-\psi^{0}, E(t)\right)$. To simplify the notation we denote

$$
\tilde{f}^{-}(t)=\left(f^{-}(t)-\mu^{-}\right)+\partial_{v_{2}} \mu^{-}\left(\psi(t)-\psi^{0}\right), \quad \tilde{\psi}(t)=\psi(t)-\psi^{0} .
$$

Define $\tilde{f}_{m}^{-}(t)=\sum_{i=1}^{m}\left|\mu_{e}^{-}\right| p_{i}(t) \zeta_{i}$ with $p_{i}(t)=\int_{0}^{P} \int_{\mathbb{R}^{2}} \zeta_{i} \tilde{f}^{-}(t) d v d x$. Then estimate (2.9) can be written as

$$
\left|p_{i}\right|(t) \leq C\left(d(0)^{\frac{1}{2}}+d(0)+d(t)\right) .
$$


So

Now by definition, $\tilde{\mathscr{P}}_{m}^{-}\left(\hat{v}_{2} \tilde{\psi}\right)(t)=\sum_{i=1}^{m} q_{i}(t) \zeta_{i}$ where $q_{i}(t)=\int_{0}^{P} \int_{\mathbb{R}^{2}}\left|\mu_{e}^{-}\right| \zeta_{i} \hat{v}_{2} \tilde{\psi}(t) d v d x$.

$$
\left|q_{i}\right|(t) \leq C|\tilde{\psi}|_{\infty}(t) \leq C\|\tilde{\psi}\|_{H^{1}}(t) \leq C d(t)^{\frac{1}{2}}
$$

We write

$$
\int_{0}^{P} \int_{\mathbb{R}^{2}} \tilde{\mathscr{P}}_{m}^{-}\left(\hat{v}_{2} \tilde{\psi}\right) \tilde{f}^{-} d x d v=b_{1}+b_{2}
$$

where

$$
\begin{aligned}
& b_{1}=\int_{0}^{P} \int_{\mathbb{R}^{2}} \tilde{\mathscr{P}}_{m}^{-}\left(\hat{v}_{2} \tilde{\psi}\right) \tilde{f}_{m}^{-} d x d v \\
& b_{2}=\int_{0}^{P} \int_{\mathbb{R}^{2}} \tilde{\mathscr{P}}_{m}^{-}\left(\hat{v}_{2} \tilde{\psi}\right)\left(\tilde{f}^{-}-\tilde{f}_{m}^{-}\right) d x d v
\end{aligned}
$$

Since $\left\{\zeta_{1}, \zeta_{2}, \cdots, \zeta_{m}\right\}$ is an orthonormal basis in $L_{\left|\mu_{e}^{-}\right|}^{2}$, we have

$$
\begin{aligned}
\left|b_{1}(t)\right| & =\left|\int_{0}^{P} \int_{\mathbb{R}^{2}}\right| \mu_{e}^{-}\left|\left(\sum_{i=1}^{m} q_{i} \zeta_{i}\right)\left(\sum_{i=1}^{m} p_{i} \zeta_{i}\right) d x d v\right| \\
& =\left|\sum_{i=1}^{m} p_{i} q_{i}\right| \leq C\left(d(0)^{\frac{1}{2}}+d(0)+d(t)\right) d(t)^{\frac{1}{2}}
\end{aligned}
$$

and

$$
\begin{aligned}
b_{2}(t) & =\sum_{i=1}^{m} q_{i}(t) \int_{0}^{P} \int_{\mathbb{R}^{2}} \zeta_{i}\left(\tilde{f}^{-}(t)-\sum_{j=1}^{m} p_{i}(t)\left|\mu_{e}^{-}\right| \zeta_{j}\right) d v d x \\
& =\sum_{i=1}^{m} q_{i}\left(\int_{0}^{P} \int_{\mathbb{R}^{2}} \zeta_{i} \tilde{f}^{-} d v d x-p_{i}\right)=0 .
\end{aligned}
$$

So

$$
\left|\int_{0}^{P} \int_{\mathbb{R}^{2}} \tilde{\mathscr{P}}_{m}^{-}\left(\hat{v}_{2} \tilde{\psi}\right) \tilde{f}^{-} d x d v\right| \leq C\left(d(0)^{\frac{1}{2}}+d(0)+d(t)\right) d(t)^{\frac{1}{2}}
$$

\subsection{Duality transformation of nonlinear terms}

Now we estimate $I\left(f^{-}, \psi, E\right)-I\left(\mu^{-}, \psi^{0}, 0\right)$. We split it in the same way as before: $I=I_{1}+I_{2}+I_{3}$. But then we further split $I_{1}$ in a rather delicate way. The estimate for the term $I_{2}$ is the same as before. We write $I_{1}=I_{1}^{-}+I_{1}^{+}$and rewrite the term $I_{1}^{-}$as follows, using the notation $\tilde{\psi}=\psi-\psi^{0}$. A free parameter $\tau$ will be 
chosen later.

$$
\begin{aligned}
I_{1}^{-}= & d_{1}^{-}\left(f^{-}, \mu^{-}\right)-\int_{0}^{P} \int_{\mathbb{R}^{2}}\left(\eta\left(v_{2}-\psi\right)-\eta\left(v_{2}-\psi^{0}\right)\right)\left(f^{-}-\mu^{-}\right) d x d v \\
= & \tau d_{1}^{-}\left(f^{-}, \mu^{-}\right)+\tau \int_{0}^{P} \int_{\mathbb{R}^{2}} \eta^{\prime}\left(v_{2}-\psi^{0}\right)(\tilde{\psi})\left(f^{-}-\mu^{-}\right) d v d x \\
& +(1-\tau)\left(d_{1}^{-}\left(f^{-}, \mu^{-}\right)+\int_{0}^{P} \int_{\mathbb{R}^{2}} \eta^{\prime}\left(v_{2}-\psi^{0}\right) \tilde{\psi}\left(\tilde{f}^{-}-\partial_{v_{2}} \mu^{-} \tilde{\psi}\right) d v d x\right) \\
& -\int_{0}^{P} \int_{\mathbb{R}^{2}}\left(\eta\left(v_{2}-\psi\right)-\eta\left(v_{2}-\psi^{0}\right)+\eta^{\prime}\left(v_{2}-\psi^{0}\right) \tilde{\psi}\right)\left(f^{-}-\mu^{-}\right) d x d v .
\end{aligned}
$$

We substitute $\eta^{\prime}=\hat{v}_{2}+\partial_{v_{2}} \mu^{-} / \mu^{-}$to get

$$
\begin{aligned}
I_{1}^{-}= & \tau d_{1}^{-}\left(f^{-}, \mu^{-}\right)+\tau \int_{0}^{P} \int_{\mathbb{R}^{2}} \eta^{\prime}\left(v_{2}-\psi^{0}\right)\left(\psi-\psi^{0}\right)\left(f^{-}-\mu^{-}\right) d v d x \\
& +(1-\tau)\left(d_{1}^{-}\left(f^{-}, \mu^{-}\right)+\int_{0}^{P} \int_{\mathbb{R}^{2}}\left(\left(1-\tilde{\mathscr{P}}_{m}^{-}\right)\left[\hat{v}_{2} \tilde{\psi}\right] \tilde{f}^{-}+\frac{\partial_{v_{2}} \mu^{-}}{\mu^{-}} \tilde{\psi} \tilde{f}^{-}\right) d v d x\right) \\
& +(1-\tau) \int_{0}^{P} \int_{\mathbb{R}^{2}} \tilde{\mathscr{P}}_{m}^{-}\left[\hat{v}_{2} \tilde{\psi}\right] \tilde{f}^{-} d v d x-(1-\tau) \int_{0}^{P} \int_{\mathbb{R}^{2}} \eta^{\prime}\left(v_{2}-\psi^{0}\right) \partial_{v_{2}} \mu^{-} \tilde{\psi}^{2} d v d x \\
& -\int_{0}^{P} \int_{\mathbb{R}^{2}}\left(\eta\left(v_{2}-\psi\right)-\eta\left(v_{2}-\psi^{0}\right)+\eta^{\prime}\left(v_{2}-\psi^{0}\right) \tilde{\psi}\right)\left(f^{-}-\mu^{-}\right) d x d v \\
\equiv & \sum_{i=1}^{5} I_{1, i}^{-}
\end{aligned}
$$

where $0<\tau<1$ is free to be determined. In these calculations we wrote

$$
\eta^{\prime}\left(v_{2}-\psi^{0}\right) \tilde{\psi}=\left(1-\tilde{\mathscr{P}}_{m}^{-}\right)\left[\hat{v}_{2} \tilde{\psi}\right]+\tilde{\mathscr{P}}_{m}^{-}\left[\hat{v}_{2} \tilde{\psi}\right]+\frac{\partial_{v_{2}} \mu^{-}}{\mu^{-}} \tilde{\psi} .
$$

Now we estimate each of these terms separately. By (2.8), we have

$$
\begin{aligned}
I_{1,1}^{-} & \geq \tau d_{1}^{-}\left(f^{-}, \mu^{-}\right)-C \tau\left\|\eta^{\prime}\right\|_{L^{\infty}}\left\|\psi-\psi^{0}\right\|_{L^{\infty}} d_{1}^{-}\left(f^{-}, \mu^{-}\right)^{\frac{1}{2}} \\
& \geq \frac{1}{2} \tau d_{1}^{-}\left(f^{-}, \mu^{-}\right)-C^{\prime} \tau\left\|\psi-\psi^{0}\right\|_{H^{1}}^{2} .
\end{aligned}
$$

By (2.10),

$$
\left|I_{1,3}^{-}\right| \leq C\left(d(0)^{\frac{1}{2}}+d(0)+d(t)\right) d(t)^{\frac{1}{2}} .
$$

By the mean value theorem and using (2.8) again, we have

$$
\left|I_{1,5}^{-}\right| \leq C\left|\eta^{\prime \prime}\right|_{\infty}\left\|\psi-\psi^{0}\right\|_{H^{1}}^{2} d_{1}^{-}\left(f^{-}, \mu^{-}\right)^{\frac{1}{2}} \geq C^{\prime \frac{3}{2}} .
$$

To estimate the second term, we use Lemma 2.1 by setting

$$
f_{0}=\mu^{-}, f=\tilde{f}^{-}, h=-\left(1-\tilde{\mathscr{P}}_{m}^{-}\right)\left(\hat{v}_{2} \tilde{\psi}\right), c=-\frac{\partial_{v_{2}} \mu^{-}}{\mu^{-}} \tilde{\psi}, d=-\partial_{v_{2}} \mu^{-} \tilde{\psi}
$$


20

Z. LIN AND W. STRAUSS

so that

$$
\begin{aligned}
I_{1,2}^{-} & =(1-\tau) \iint\left\{H\left(\tilde{f}^{-}+\mu^{-}-\partial_{v_{2}} \mu^{-} \tilde{\psi}\right)-H\left(\mu^{-}\right)-H^{\prime}\left(\mu^{-}\right)\left(\tilde{f}^{-}-\partial_{v_{2}} \mu^{-} \tilde{\psi}\right)\right. \\
& \left.+\left(1-\tilde{\mathscr{P}}_{m}^{-}\right)\left(\hat{v}_{2} \tilde{\psi}\right) \tilde{f}^{-}+\frac{\partial_{v_{2}} \mu^{-}}{\mu^{-}} \tilde{\psi} \tilde{f}^{-}\right\} d v d x .
\end{aligned}
$$

By Lemma 2.1,

$$
\begin{aligned}
I_{1,2}^{-} & \geq(1-\tau) \iint \mu^{-}\left\{1-\left(1-\tilde{\mathscr{P}}_{m}^{-}\right)\left(\hat{v}_{2} \tilde{\psi}\right)-\frac{\partial_{v_{2}} \mu^{-}}{\mu^{-}} \tilde{\psi}\right\} \\
& -\mu^{-} \exp \left[-\left(1-\tilde{\mathscr{P}}_{m}^{-}\right)\left(\hat{v}_{2} \tilde{\psi}\right)-\frac{\partial_{v_{2}} \mu^{-}}{\mu^{-}} \tilde{\psi}\right] \\
& +\left[\left(1-\tilde{\mathscr{P}}_{m}^{-}\right)\left(\hat{v}_{2} \tilde{\psi}\right)+\frac{\partial_{v_{2}} \mu^{-}}{\mu^{-}} \tilde{\psi}\right] \partial_{v_{2}} \mu^{-} \tilde{\psi}
\end{aligned}
$$

Now we do the Taylor expansion of $1+\xi-e^{\xi}$ for

$$
c+h=\xi=-\left(1-\tilde{\mathscr{P}}_{m}^{-}\right)\left(\hat{v}_{2} \tilde{\psi}\right)-\frac{\partial_{v_{2}} \mu^{-}}{\mu^{-}} \tilde{\psi}
$$

and use the estimate

$$
|\xi|=\left|\left(1-\tilde{\mathscr{P}}_{m}^{-}\right)\left(\hat{v}_{2} \tilde{\psi}\right)+\frac{\partial_{v_{2}} \mu^{-}}{\mu^{-}} \tilde{\psi}\right| \leq C|\tilde{\psi}|_{\infty} \leq C^{\prime}\left\|\psi-\psi^{0}\right\|_{H^{1}} .
$$

Therefore

$$
\begin{aligned}
I_{1,2}^{-} & \geq(1-\tau) \iint\left\{-\frac{1}{2} \mu^{-}\left[\left(1-\tilde{\mathscr{P}}_{m}^{-}\right)\left(\hat{v}_{2} \tilde{\psi}\right)+\frac{\partial_{v_{2}} \mu^{-}}{\mu^{-}} \tilde{\psi}\right]^{2}\right. \\
& \left.+\left[\left(1-\tilde{\mathscr{P}}_{m}^{-}\right)\left(\hat{v}_{2} \tilde{\psi}\right)+\frac{\partial_{v_{2}} \mu^{-}}{\mu^{-}} \tilde{\psi}\right] \partial_{v_{2}} \mu^{-} \tilde{\psi}\right\} d v d x-C e^{C^{\prime}\|\tilde{\psi}\|_{H^{1}}}\|\tilde{\psi}\|_{H^{1}}^{3} .
\end{aligned}
$$

Now we use $\partial_{v_{2}} \mu^{-} / \mu^{-}=-\hat{v}_{2}+\eta^{\prime}$ and

$$
\left(1-\tilde{\mathscr{P}}_{m}^{-}\right)\left(\hat{v}_{2} \tilde{\psi}\right)+\frac{\partial_{v_{2}} \mu^{-}}{\mu^{-}} \tilde{\psi}=-\tilde{\mathscr{P}}_{m}^{-}\left(\hat{v}_{2} \tilde{\psi}\right)+\eta^{\prime} \tilde{\psi}
$$

to get

$$
\begin{aligned}
I_{1,2}^{-} & \geq(1-\tau) \iint\left\{\left(\frac{1}{2} \mu^{-}\left[\eta^{\prime}\left(v_{2}-\psi^{0}\right)\right]^{2}-\mu^{-} \hat{v}_{2} \eta^{\prime}\left(v_{2}-\psi^{0}\right)\right) \tilde{\psi}^{2}\right. \\
& \left.+\frac{1}{2} \mu^{-}\left(\tilde{\mathscr{P}}_{m}^{-}\left[\hat{v}_{2} \tilde{\psi}\right]\right)^{2}\right\} d v d x-C e^{C^{\prime}\|\tilde{\psi}\|_{H^{1}}}\|\tilde{\psi}\|_{H^{1}}^{3} .
\end{aligned}
$$

Also

$$
I_{1,4}=(1-\tau) \iint\left[\eta^{\prime} \hat{v}_{2} \mu \tilde{\psi}^{2}-\mu\left(\eta^{\prime 2} \tilde{\psi}^{2}\right] d v d x\right.
$$


Combining all these estimates, we get

$$
\begin{aligned}
I_{1}^{-}(t) & \geq \frac{1}{2} \tau d_{1}^{-}\left(f^{-}, \mu^{-}\right) \\
& +(1-\tau) \iint\left(-\frac{1}{2} \mu^{-} \eta^{\prime}\left(v_{2}-\psi^{0}\right)^{2} \tilde{\psi}^{2}+\frac{1}{2} \mu^{-}\left(\tilde{\mathscr{P}}_{m}^{-}\left[\hat{v}_{2} \tilde{\psi}\right]\right)^{2}\right) d v d x \\
& -C e^{C^{\prime} d(t)^{\frac{1}{2}}} d(t)^{\frac{3}{2}}-C^{\prime} \tau\|\tilde{\psi}\|_{H^{1}}^{2}-C d(t)^{\frac{3}{2}}-C\left(d(0)^{\frac{1}{2}}+d(0)\right) d(t)^{\frac{1}{2}},
\end{aligned}
$$

recalling that $d(t) \geq\|\tilde{\psi}(t)\|_{H^{1}}^{2}$. Similarly, we have

$$
\begin{aligned}
I_{1}^{+}(t) & \geq \frac{1}{2} \tau d_{1}^{+}\left(f^{+}, \mu^{+}\right) \\
& +(1-\tau) \int_{0}^{P} \int_{\mathbb{R}^{2}}\left\{-\frac{1}{2} \mu^{+}\left[\eta^{\prime}\left(v_{2}+\psi^{0}\right)\right]^{2} \tilde{\psi}^{2}+\frac{1}{2} \mu^{+}\left(\tilde{\mathscr{P}}_{m}^{+}\left[\hat{v}_{2} \tilde{\psi}\right]\right)^{2}\right\} d v d x \\
& -C e^{C^{\prime} d(t)^{\frac{1}{2}}} d(t)^{\frac{3}{2}}-C^{\prime} \tau\|\tilde{\psi}\|_{H^{1}}^{2}-C d(t)^{\frac{3}{2}}-C\left(d(0)^{\frac{1}{2}}+d(0)\right) d(t)^{\frac{1}{2}} .
\end{aligned}
$$

We remark that in contrast to the straightforward estimate (2.5), the constrained energy-Casimir method gives us the additional stabilizing term $\iint \mu^{ \pm} \tilde{\mathscr{P}}_{m}^{ \pm}\left[\hat{v}_{2} \tilde{\psi}\right]^{2} d v d x$, which is crucial for the sharp nonlinear stability criterion.

\subsection{Proof of orbital stability}

Combining the preceding estimates with that of $I_{2}$ (see (2.4)) and the definition of $I_{3}$, we have

$$
\begin{aligned}
& I\left(f^{ \pm}, \psi, E\right)-I\left(\mu^{ \pm}, \psi^{0}, 0\right) \\
& \geq \frac{1}{2} \tau d_{1}^{ \pm}\left(f^{ \pm}, \mu^{ \pm}\right)+\left(\tilde{\mathscr{L}}_{m}\left(\psi-\psi^{0}\right),\left(\psi-\psi^{0}\right)\right)+\frac{1}{2}\|E\|_{2}^{2}-C^{\prime} \tau\left\|\psi-\psi^{0}\right\|_{H^{1}}^{2} \\
& \quad-C e^{C^{\prime} d(t)^{\frac{1}{2}}} d(t)^{\frac{3}{2}}-C d(t)^{\frac{3}{2}}-C\left(d(0)^{\frac{1}{2}}+d(0)\right) d(t)^{\frac{1}{2}}
\end{aligned}
$$

recalling the definition of $\tilde{\mathscr{L}}_{m}$ by (2.6) and throwing away the extra term

$$
\frac{\tau}{2} \iint \sum_{ \pm} \mu\left(\eta^{\prime}\right)^{2} \tilde{\psi}^{2} d v d x \geq 0
$$

Now we use translation to get rid of $\operatorname{ker} \tilde{\mathscr{L}}_{m}=\left\{\psi_{x}^{0}\right\}$. We choose $\theta(t)$ so that

$$
\left\|\psi(t)-T_{\theta(t)} \psi^{0}\right\|_{2}=\min _{\theta}\left\|\psi(t)-T_{\theta} \psi^{0}\right\|_{2}
$$

which implies that

$$
\left(\psi(t)-T_{\theta(t)} \psi^{0}, T_{\theta(t)} \psi_{x}^{0}\right)=\left(T_{-\theta(t)} \psi(t)-\psi^{0}, \psi_{x}^{0}\right)=0 .
$$

Next we replace $\left(f^{ \pm}, \psi, E\right)(t)$ by $T_{-\theta(t)}\left(\left(f^{ \pm}, \psi, E\right)(t)\right)$. Since all the functionals $I\left(f^{ \pm}, \psi, E\right)$ and $J_{i}\left(f^{-}, \psi\right)$ are invariant under the translation, we can apply all the 
estimates to the translated functions to get

$$
\begin{aligned}
& I\left(T_{-\theta(t)} f^{ \pm}, T_{-\theta(t)} \psi, T_{-\theta(t)} E\right)-I\left(\mu^{ \pm}, \psi^{0}, 0\right) \\
& \geq \frac{1}{2} \tau d_{1}^{ \pm}\left(T_{-\theta(t)} f^{ \pm}, \mu^{ \pm}\right)+\frac{1}{2}\left(\tilde{\mathscr{L}}_{m}\left(T_{-\theta(t)} \psi-\psi^{0}\right),\left(T_{-\theta(t)} \psi-\psi^{0}\right)\right) \\
& +\frac{1}{2}\|E\|_{2}^{2}-C^{\prime} \tau\left\|T_{-\theta(t)} \psi-\psi^{0}\right\|_{H^{1}}^{2}-C e^{C^{\prime} d(t)^{1 / 2}} d(t)^{\frac{3}{2}} \\
& -C d(t)^{\frac{3}{2}}-C\left(d(0)^{\frac{1}{2}}+d(0)\right) d(t)^{\frac{1}{2}}
\end{aligned}
$$

with

$$
d(t)=\left\|T_{-\theta(t)} \psi-\psi^{0}\right\|_{H^{1}}^{2}+\left\|T_{-\theta(t)} E\right\|_{2}^{2}+\sum_{ \pm} d_{1}^{ \pm}\left(T_{-\theta(t)} f^{ \pm}, \mu^{ \pm}\right) .
$$

From $\tilde{\mathscr{L}}_{m}>0$ on $\left\{\psi_{x}^{0}\right\}^{\perp}$, it follows that there exists $c_{0}>0$ such that $\left(\tilde{\mathscr{L}}_{m} \phi, \phi\right) \geq$ $c_{0}\|\phi\|_{H^{1}}^{2}$ for any $\phi \in H^{1}$ and $\left(\phi, \psi_{x}^{0}\right)=0$. Choosing $\tau$ so that $C^{\prime} \tau \leq \frac{1}{2} c_{0}$, we have

$$
\begin{aligned}
& I\left(f^{ \pm}(0), \psi(0), E(0)\right)-I\left(\mu^{ \pm}, \psi^{0}, 0\right) \\
& =I\left(T_{-\theta(t)} f^{ \pm}, T_{-\theta(t)} \psi, T_{-\theta(t)} E\right)-I\left(\mu^{ \pm}, \psi^{0}, 0\right) \\
& \geq c_{1} d(t)-C e^{C^{\prime} d(t)^{1 / 2}} d(t)^{\frac{3}{2}}-C d(t)^{\frac{3}{2}}-C\left(d(0)^{\frac{1}{2}}+d(0)\right) d(t)^{\frac{1}{2}}
\end{aligned}
$$

for some $c_{1}>0$. It is easy to see that

$$
I\left(f^{ \pm}(0), \psi(0), E(0)\right)-I\left(\mu^{ \pm}, \psi^{0}, 0\right) \leq C^{\prime \prime} d(0)
$$

for some $C^{\prime \prime}>0$.

Writing $x=d(t)^{1 / 2}$, we define the functions $y_{1}=c_{1} x^{2}-C e^{C^{\prime} x} x^{3}-C x^{3}$ and $y_{2}=C\left(d(0)^{1 / 2}+d(0)\right) x+C^{\prime \prime} d(0)$. Then (2.11) implies that $y_{1}\left(d(t)^{1 / 2}\right) \leq$ $y_{2}\left(d(t)^{1 / 2}\right)$. Now $y_{1}(x)$ has a single positive maximum $y\left(x_{0}\right)$, is increasing in $\left(0, x_{0}\right)$, is decreasing in $\left(x_{0}, \infty\right)$ and tends to $-\infty$. So it is obvious that if $d(0)$ is sufficiently small, the line $y=y_{2}(x)$ intersects the curve $y=y_{1}(x)$ at exactly two points $x_{1}(d(0))<x_{0}<x_{2}(d(0))$. There are two disjoint intervals $\left[0, x_{1}(d(0))\right]$ and $\left[x_{2}(d(0)), \infty\right)$ such that $y_{1}(x) \leq y_{2}(x)$. Since $d(t)$ is continuous, we deduce $d(t)^{1 / 2}<x_{1}(d(0))$ for all $t<\infty$, provided we choose $d(0)^{1 / 2}<x_{0}$. Since $x_{1}(d(0)) \rightarrow 0$ as $d(0) \rightarrow 0$, we deduce the nonlinear stability in terms of the distance functional $d(t)^{1 / 2}$. By (2.8) this implies the stability in the sense of (1.7) and completes the proof of Theorem 1.2.

\section{Nonlinear instability of one and one half RVM}

For simplicity, we consider a periodic equilibrium with a fixed ion background. The proof can easily be carried over to the two-species case. The nonlinear $1 \frac{1}{2}$ RVM in the one-species case is (with $f=f^{-}$)

$$
\partial_{t} f+\hat{v}_{1} \partial_{x} f-\left(E_{1}+\hat{v}_{2} B\right) \partial_{v_{1}} f-\left(E_{2}-\hat{v}_{1} B\right) \partial_{v_{2}} f=0
$$




$$
\begin{gathered}
\partial_{t} E_{1}=-j_{1}=\int \hat{v}_{1} f d v, \partial_{t} B=-\partial_{x} E_{2} \\
\partial_{t} E_{2}+\partial_{x} B=-j_{2}=\int \hat{v}_{2} f d v
\end{gathered}
$$

with the constraint

$$
\partial_{x} E_{1}=n_{0}-\int f d v
$$

We consider an equilibrium of the form $\left(f^{0}=\mu(e, p), E_{1}^{0}, B^{0}\right)$ where $e=\sqrt{1+v_{1}^{2}+v_{2}^{2}}-$ $\phi^{0}(x), p=v_{2}-\psi^{0}(x), E_{1}^{0}=-\phi^{0 \prime}(x), B^{0}(x)=\psi^{0 \prime}(x)$ with $\psi^{0}$ and $\phi^{0}$ satisfying the pair of equations

$$
\begin{aligned}
-\phi^{0 \prime \prime} & =n_{0}-\int \mu(e, p) d v \\
\psi^{0 \prime \prime} & =\int \hat{v}_{2} \mu(e, p) d v .
\end{aligned}
$$

In this section we assume there exists a growing mode solution $e^{\lambda t}\left(f_{g}, E_{g 1}, E_{g 2}, B_{g}\right)$ of the linearized system

(3.2)

$$
\begin{gathered}
\partial_{t} f+\hat{v}_{1} \partial_{x} f-\left(E_{1}^{0}+\hat{v}_{2} B^{0}\right) \partial_{v_{1}} f+\hat{v}_{1} B^{0} \partial_{v_{2}} f=\left(E_{1}+\hat{v}_{2} B\right) \partial_{v_{1}} f^{0}+\left(E_{2}-\hat{v}_{1} B\right) \partial_{v_{2}} f^{0} \\
\partial_{t} E_{1}=\int \hat{v}_{1} f d v, \partial_{t} B=-\partial_{x} E_{2} \\
\partial_{t} E_{2}+\partial_{x} B=\int \hat{v}_{2} f d v
\end{gathered}
$$

with the constraint

$$
\partial_{x} E_{1}=-\int f d v
$$

The main result of this section is the following theorem, which is essentially the same as Theorem 1 .

Theorem 3.1. Let $\left(f^{0}, E_{1}^{0}, B^{0}\right)$ a periodic equilibrium solution of (3.1d) of the form given above with $E_{2}^{0}=0$ such that

$$
\mu=O\left(\langle v\rangle^{-\eta}\right), \eta>3,\left|\mu_{e}\right|+\left|\mu_{p}\right|=O(\mu), \sup _{x} \int\langle v\rangle^{2}\left(\left|\mu_{e}\right|+\left|\mu_{p}\right|\right) d v<\infty .
$$

If there exists a growing mode with $f_{g} \in L^{1}$ and $E, B \in W^{1,1}$, then $f_{g} \in W^{1,1}$ and there exist positive constants $\varepsilon_{0}, C_{1}$ and a family of $W^{1,1}$ solutions $\left[\bar{f}^{\delta}, \bar{E}_{1}^{\delta}, \bar{E}_{2}^{\delta}, \bar{B}^{\delta}\right]$ of (3.1d) of period $P$ in $x$, defined for $\delta$ sufficiently small, with $\bar{f}^{\delta}(t)$ non-negative, such that

$$
\left\|\bar{f}^{\delta}(0)-f^{0}\right\|_{W^{1,1}([0, P] \times \mathbb{R})}+\left\|\bar{E}^{\delta}(0)-E^{0}\right\|_{W^{1,1}(0, P)}+\left\|\bar{B}^{\delta}(0)-B^{0}\right\|_{W^{1,1}(0, P)} \leq \delta
$$

and

$$
\sup _{0 \leq t \leq C_{1}|\ln \delta|}\left\|\bar{E}^{\delta}(t)-E^{0}\right\|_{L^{1}(0, P)}+\left\|\bar{B}^{\delta}(t)-B^{0}\right\|_{L^{1}(0, P)} \geq \varepsilon_{0}
$$


The pointwise decay hypothesis on $\mu$ is made only to assure well-posedness. The hypothesis $\left|\mu_{e}\right|+\left|\mu_{p}\right|=O(\mu)$ is used only to assure that the unstable solution has non-negative density. We divide the proof of the theorem into several steps.

\subsection{Averaging Liapunov exponent and properties of growing modes}

The particle trajectory equation is

$$
\left\{\begin{array}{c}
\dot{X}=\hat{V}_{1} \\
\dot{V}_{1}=-\left(E_{1}^{0}(X)+\hat{V}_{2} B^{0}(X)\right) \\
\dot{V}_{2}=\hat{V}_{1} B^{0}(X) .
\end{array}\right.
$$

We want to understand the properties of the Jacobian matrix $J(t)=\frac{\partial\left(X, V_{1}, V_{2}\right)}{\partial\left(x, v_{1}, v_{2}\right)}(t)$. We have

Lemma 3.2. (i) For any $\alpha>0$, there exist constants $M_{\alpha}, C_{\alpha}$ such that if $\left|v_{1}\right|+$ $\left|v_{2}\right|>M_{\alpha}$ then $|J(t)|_{\infty} \leq C_{\alpha}^{1} e^{\alpha t}$.

(ii) For any positive constants $M$ and $\alpha$, there exists a constant $C_{\alpha}^{2}$ such that

$$
\iint_{\left|v_{1}\right|+\left|v_{2}\right| \leq M}|J(t)|_{2} d v d x \leq C_{\alpha}^{2} e^{\alpha t} .
$$

Here we use the usual matrix norms for an $n \times n$ matrix $A$, namely, that $|A|_{\infty}$ denotes the maximum of the entries and $|A|_{2}$ denotes the $\ell^{2}$-norm of the entries.

Proof. The proof of (i) is easy. We have

$$
\begin{aligned}
\frac{\partial J}{\partial t} & =\left(\begin{array}{ccc}
0 & \frac{V_{2}^{2}+1}{\langle V\rangle^{3}} & -\frac{V_{1} V_{2}}{\langle V\rangle^{3}} \\
-\partial_{x} E_{1}^{0}(X)-\hat{V}_{2} \partial_{x} B^{0}(X) & \frac{V_{1} V_{2}}{\langle V\rangle^{3}} B^{0}(X) & -\frac{V_{1}^{2}+1}{\langle V\rangle^{3}} B^{0}(X) \\
\hat{V}_{1} \partial_{x} B^{0}(X) & \frac{V_{1}^{2}+1}{\langle V\rangle^{3}} B^{0}(X) & -\frac{V_{1} V_{2}}{\langle V\rangle^{3}} B^{0}(X)
\end{array}\right) J \\
& =H(X, V) J .
\end{aligned}
$$

When $\left|v_{1}\right|+\left|v_{2}\right|=M$ is large, the matrix $H(t)$ is very close to

$$
H^{\theta}=\left(\begin{array}{ccc}
0 & 0 & 0 \\
-\partial_{x} E_{1}^{0}(X)-\cos \theta \partial_{x} B^{0}(X) & 0 & 0 \\
\sin \theta \partial_{x} B^{0}(X) & 0 & 0
\end{array}\right)
$$

for some $\theta$, uniformly in $M$. Since $H^{\theta}$ only has eigenvalues 0 , when $M$ is large all the eigenvalues of $H$ have very small real part. Thus the conclusion of (i) follows by standard ODE theory.

The proof of (ii) is similar to the proof of the corresponding result in the 2D Euler case ([13]), so we only sketch it. Since $V_{2}(t)+\psi^{0}(X)=v_{2}+\psi^{0}(x)$, we have

$$
\frac{\partial V_{2}}{\partial x}=\partial_{x} \psi^{0}(x)-\partial_{x} \psi^{0}(X) \frac{\partial X}{\partial x}, \frac{\partial V_{2}}{\partial v_{1}}=-\partial_{x} \psi^{0}(X) \frac{\partial X}{\partial v_{1}}, \frac{\partial V_{2}}{\partial v_{2}}=1-\partial_{x} \psi^{0}(X) \frac{\partial X}{\partial v_{2}} .
$$


So we only need to estimate the $L^{1}$-norm of the derivative of $X$ and $V_{1}$. We use the new variable $p=v_{2}+\psi^{0}(x)$ instead of $v_{2}$; note that this does not change the conclusion. For each fixed $p$, the particle motion is described by the reduced system

$$
\left\{\begin{array}{c}
X=\partial_{v_{1}} H_{p} \\
V_{1}=-\partial_{x} H_{p}
\end{array}\right.
$$

where $H_{p}\left(x, v_{1}\right)=\sqrt{1+v_{1}^{2}+\left(p-\psi^{0}(x)\right)^{2}}-\phi^{0}(x)=e$ is the energy. The critical point of $(3.5)$ is $\left(x, v_{1}\right)=\left(x_{0}(p), 0\right)$ where $x_{0}(p)$ solves the equation

$$
E_{1}^{0}\left(x_{0}\right)+\frac{p-\psi^{0}\left(x_{0}\right)}{\sqrt{1+\left(p-\psi^{0}\left(x_{0}\right)\right)^{2}}} B^{0}\left(x_{0}\right)=0 .
$$

The critical energy level is $e_{c}(p)=\sqrt{1+\left(p-\psi^{0}\left(x_{0}(p)\right)\right)^{2}}-\phi^{0}\left(x_{0}(p)\right)$. If $e \neq$ $e_{c}(p)$, then the particle executes periodic motion with period $T(e, p)$. If $e=e_{c}(p)$, then the particle is either in equilibrium or travels along a trajectory connecting saddle points. Let

$$
A_{M}=\left\{\left(x, v_{1}, p\right) \in[0, P] \times[-M, M] \times\left[-M+\min \psi^{0}, M+\max \psi^{0}\right]\right\} .
$$

The "dangerous set" $A_{\mathrm{cr}} \subset A_{M}$ is the collection of nondegenerate saddle points of $H_{p}$ and the trajectories connecting them, for all $p \in\left[-M+\min \psi^{0}, M+\max \psi^{0}\right]$.

Let $A^{\varepsilon}$ be the $\varepsilon$-neighborhood of $A_{\mathrm{cr}}$ in $A_{M}$. On $A_{M} \backslash A^{\varepsilon}$, the Liapunov exponent of (3.4) is zero, so for any $\alpha>0$ there exists $C_{a}^{\prime}$ such that $|J(t)|_{2} \leq C_{\alpha}^{\prime} e^{\alpha t}$ thus

$$
\int_{A_{M} \backslash A^{\varepsilon}}|J(t)|_{2} d v d x \leq\left|A_{M} \backslash A^{\varepsilon}\right| C_{\alpha}^{\prime} e^{\alpha t}
$$

Now we estimate the integral in the set $A^{\varepsilon}$. For fixed $x, v_{1}$, denote $I_{p}^{x, v_{1}}=\left\{p \mid\left(x, v_{1}, p\right) \in A^{\varepsilon}\right\}$ and similarly denote $I_{v_{1}}^{x, p}$ and $I_{x}^{p, v_{1}}$. Then

$$
\begin{aligned}
& \iiint_{A^{\varepsilon}} \sqrt{\left|\frac{\partial X}{\partial x}\right|^{2}+\left|\frac{\partial V_{1}}{\partial x}\right|} d x d v=\int d p \int d v_{1} \int_{I_{x}^{p, v_{1}}} \sqrt{\left|\frac{\partial X}{\partial x}\right|^{2}+\left|\frac{\partial V_{1}}{\partial x}\right|} d x \\
& \iiint_{A^{\varepsilon}} \sqrt{\left|\frac{\partial X}{\partial v_{1}}\right|^{2}+\left|\frac{\partial V_{1}}{\partial v_{1}}\right|} d x d v=\int d p \int d x \int_{I_{v_{1}, p}} \sqrt{\left|\frac{\partial X}{\partial v_{1}}\right|^{2}+\left|\frac{\partial V_{1}}{\partial v_{1}}\right|} d v_{1} \\
& \iiint_{A^{\varepsilon}} \sqrt{\left|\frac{\partial X}{\partial p}\right|^{2}+\left|\frac{\partial V_{1}}{\partial p}\right|} d x d v=\int d v_{1} \int d x \int_{I_{p}^{x, v_{1}}} \sqrt{\left|\frac{\partial X}{\partial p}\right|^{2}+\left|\frac{\partial V_{1}}{\partial p}\right|} d p
\end{aligned}
$$

In these three expressions the three innermost integrals on the right sides are the lengths of the planar curves

$$
\begin{gathered}
l^{x}(t): x \in I_{x}^{p, v_{1}} \rightarrow(X(t, x), V(t, x)) \text { for fixed } p, v_{1} ; \\
l^{v_{1}}(t): v_{1} \in I_{v_{1}}^{x, p} \rightarrow\left(X\left(t, v_{1}\right), V\left(t, v_{1}\right)\right) \text { for fixed } p, x ;
\end{gathered}
$$




$$
l^{p}(t): p \in I_{p}^{x, v_{1}} \rightarrow(X(t, p), V(t, p)) \text { for fixed } x, v_{1} .
$$

Here we only analyze the length of $l^{p}(t)$ since the other two are similar. We split the interval $I_{p}^{x, v_{1}}$ into certain subintervals $I_{p}^{x, v_{1}, j}=\left[p_{1}, p_{2}\right]$ such that exactly one endpoint (say $p_{1}$ ) is on $A_{\mathrm{cr}}$. The curve $l^{p}(t)$ is split into a collection of curves $l^{p, j}(t)$ in the same way. Particle 1 starting at $\left(x, v_{1}, p_{1}\right)$ approaches a saddle point in infinite time, while particle 2 starting at the other end $\left(x, v_{1}, p_{2}\right)$ has a finite period $T\left(p_{2}\right)$. If we choose $\varepsilon$ small enough, the period function $T(p)$ is monotone on $\left[p_{1}, p_{2}\right]$. At a very large time $t$, particle 1 approaches the saddle point very closely and particle 2 completes at most $\left[t / T\left(p_{2}\right)\right]+1$ periods. Thus the stretched curve $l^{p, j}(t)$ consists of at most $\left[t / T\left(p_{2}\right)\right]+1$ "circuits". If $\varepsilon$ is very small, each circuit is expected to wind tightly around the trajectory of particle 2 and thus have a comparable length $L$. So intuitively the length of $l^{p, j}(t)$ is controlled by $\left(\left[t / T\left(p_{2}\right)\right]+1\right) L$ and thus it grows only linearly in time. This implies that the integral (3.9) also grows only linearly. The rough argument just given can be made rigorous using the same procedure as in [13]. So we do not repeat it here. By the same analysis, the integrals (3.7) and (3.8) also have only linear growth. So finally we deduce

$$
\int_{A^{\varepsilon}}|J(t)|_{2} d v d x \leq C_{1} t+C_{2}
$$

for some constants $C_{1}, C_{2}$. Combined with (3.6), this implies the conclusion of (ii).

Lemma 3.3. The growing mode satisfies $f_{g} \in W^{1,1}$ and $\left|f_{g}\right| \leq C \mu$.

Proof. The proof that $f_{g} \in W^{1,1}$ is essentially the same as in the Vlasov-Poisson case (see [12]); so we merely sketch it here. Differentiating the trajectory integral formula for $f_{g}$ as in [15], we represent $\partial f_{g}$ in terms of the derivative of the fields $\left(E_{g}, B_{g}\right)$ in the almost everywhere sense. Thus to estimate $\left\|\partial f_{g}\right\|_{1}$, the key point is to show that the function

$$
q(x, v)=\left(\left|\mu_{e}\right|+\left|\mu_{p}\right|\right) \int_{-\infty}^{0} e^{\lambda s}|J(s)|\left(\left|\partial_{x} E\right|+\left|\partial_{x} B\right|\right)(X(s)) d s
$$

is integrable. To prove this, we split the $(x, v)$ space into two parts according to Lemma 3.2 and use the estimates of Lemma 3.2 to show that $q(x, v)$ is integrable. It follows that $f_{g} \in W^{1,1}$.

The proof that $f_{g}$ is pointwise bounded by a multiple of $\mu$ is similar to Lemma 7 in [9]. We present the argument here. Let $S=f_{g} / \mu$. Dividing the Vlasov equation satisfied by $f_{g}$ by $\mu$, we obtain $\lambda S+\hat{v}_{1} \partial_{x} S+m=0$, where

$$
m=\hat{v}_{1} \frac{\partial_{x} \mu}{\mu^{2}} f_{g}-\left(E_{g 1}+\hat{v}_{2} B_{g}\right) \frac{\partial_{v_{1}} \mu}{\mu}-\left(E_{g 2}-\hat{v}_{1} B_{g}\right) \frac{\partial_{v_{2}} \mu}{\mu} .
$$

Thus

$$
S=-\int_{0}^{\infty} e^{-s \hat{v}_{1} \partial_{x}} e^{-s \lambda} m d s
$$


Now $\mu=\mu\left(\langle v\rangle-\phi^{0}(x), v_{2}-\psi^{0}(x)\right)$, from which it follows that $m \in L^{\infty}$. Hence $S \in L^{\infty}$ and the proof is complete.

The hypothesis that $\left|\mu_{e}\right|+\left|\mu_{p}\right|=O(\mu)$ can be generalized as in Lemma 8 of [9].

\subsection{Representation of the field}

We write the perturbed quantities as the sum of "linear" terms and "nonlinear" terms as follows:

$$
\begin{gathered}
\bar{f}=f+f^{0}=f_{l}+f_{n}+f^{0}, \quad \bar{E}_{1}=E_{1}+E_{1}^{0}=E_{1, l}+E_{1, n}+E_{1}^{0}, \\
\bar{E}_{2}=E_{2}=E_{2, l}+E_{2, n}, \quad \bar{B}=B+B^{0}=B_{l}+B_{n}+B^{0},
\end{gathered}
$$

where

$$
\begin{gathered}
\left(\begin{array}{c}
f_{l} \\
E_{1, l} \\
E_{2, l} \\
B_{l}
\end{array}\right)(t)=e^{t \mathscr{L}}\left(\begin{array}{c}
f_{l} \\
E_{1, l} \\
E_{2, l} \\
B_{l}
\end{array}\right)(0) \\
\left(\begin{array}{c}
f_{n} \\
E_{1, n} \\
E_{2, n} \\
B_{n}
\end{array}\right)(t)=\int_{0}^{t} e^{(t-s) \mathscr{L}}\left(\begin{array}{c}
-\nabla_{v} \cdot\left(\left(E_{1}+\hat{v}_{2} B\right) f,\left(E_{1}-\hat{v}_{1} B\right) f\right)(s) \\
0 \\
0 \\
0
\end{array}\right) d s .
\end{gathered}
$$

Here the linearized operator $\mathscr{L}$ is as defined by

$$
\mathscr{L}\left(\begin{array}{c}
f \\
E_{1} \\
E_{2} \\
B
\end{array}\right)=\left(\begin{array}{c}
-D f+\hat{v}_{1} \mu_{e} E_{1}+\hat{v}_{2} \mu_{e} E_{2}-\hat{v}_{1} \mu_{p} B \\
\int \hat{v}_{1} f d v \\
\int \hat{v}_{2} f d v-\partial_{x} B \\
-\partial_{x} E_{2}
\end{array}\right)=(A+K)\left(\begin{array}{c}
f \\
E_{1} \\
E_{2} \\
B
\end{array}\right)
$$

where

$$
\begin{aligned}
A & =\left(\begin{array}{cccc}
-D & 0 & 0 & 0 \\
I_{1} & 0 & 0 & 0 \\
I_{2} & 0 & 0 & -\partial_{x} \\
0 & 0 & -\partial_{x} & 0
\end{array}\right) \\
K & =\left(\begin{array}{cccc}
0 & \hat{v}_{1} \mu_{e} & \hat{v}_{2} \mu_{e} & -\hat{v}_{1} \mu_{p} \\
0 & 0 & 0 & 0 \\
0 & 0 & 0 & 0 \\
0 & 0 & 0 & 0
\end{array}\right)
\end{aligned}
$$

with

$$
D=\hat{v}_{1} \partial_{x}-\left(E_{1}^{0}+\hat{v}_{2} B^{0}\right) \partial_{v_{1}}+\hat{v}_{1} B^{0} \partial_{v_{2}}, \quad I_{i}(g)=\int \hat{v}_{i} g d v
$$


As in [9], when $E_{2}(0)=B(0)=0$,

$$
\begin{aligned}
E_{2} & =\int_{0}^{t}\left[j_{2}(\tau, x-t+\tau)+j_{2}(\tau, x+t-\tau)\right] d \tau, \\
B & =\int_{0}^{t}\left[j_{2}(\tau, x-t+\tau)-j_{2}(\tau, x+t-\tau)\right] d \tau .
\end{aligned}
$$

For the derivatives of $E_{2}$ and $B$, we use the operator splitting idea, as follows. A typical term is

$$
\begin{aligned}
I(t, x) & =\int_{0}^{t} \partial_{x} \int \hat{v}_{2} f(\tau, x-t+\tau) d v d \tau \\
& =\int_{0}^{t} \int \frac{\hat{v}_{2}}{1-\hat{v}_{1}}\left(T^{+}-S\right) f(\tau, x-t+\tau) d v d \tau \\
& =\int \frac{\hat{v}_{2}}{1-\hat{v}_{1}}(f(t, x, v)-f(0, x-\tau, v)) d v-\int_{0}^{t} \int \frac{\hat{v}_{2}}{1-\hat{v}_{1}} S f(\tau, x-t+\tau) d v d \tau
\end{aligned}
$$

where $T^{+}=\partial_{t}+\partial_{x}$ and $S=\partial_{t}+\hat{v}_{1} \partial_{x}$. Upon using the above formulae for $E_{2, n}$ and $B_{n}$, a typical term becomes

$$
\begin{aligned}
I^{n}(t, x)= & \int \frac{\hat{v}_{2}}{1-\hat{v}_{1}}\left(f_{n}(t, x, v)-f_{n}(0, x-\tau, v)\right) d v-\int_{0}^{t} \int \frac{\hat{v}_{2}}{1-\hat{v}_{1}} S f_{n}(\tau, x-t+\tau) d v d \tau \\
= & \int \frac{\hat{v}_{2}}{1-\hat{v}_{1}} f_{n}(t, x, v) d v \\
& +\int_{0}^{t} \int \nabla v\left(\frac{\hat{v}_{2}}{1-\hat{v}_{1}}\right) \cdot\left(\left(E_{1}^{0}+\hat{v}_{2} B^{0}\right) f_{n},-\hat{v}_{1} B^{0} f_{n}\right)(\tau, x-t+\tau) d v d \tau \\
& +\int_{0}^{t} \int \nabla v\left(\frac{\hat{v}_{2}}{1-\hat{v}_{1}}\right) \cdot\left(\left(E_{1, n}+\hat{v}_{2} B_{n}\right) f^{0},\left(E_{2, n}-\hat{v}_{1} B_{n}\right) f^{0}\right)(\tau, x-t+\tau) d v d \tau \\
& +\int_{0}^{t} \int \nabla v\left(\frac{\hat{v}_{2}}{1-\hat{v}_{1}}\right) \cdot\left(\left(E_{1}+\hat{v}_{2} B\right) f,\left(E_{2}-\hat{v}_{1} B\right) f\right)(\tau, x-t+\tau) d v d \tau \\
= & I_{0}^{n}+I_{1}^{n}+I_{2}^{n}+I_{3}^{n}
\end{aligned}
$$

since

$$
\begin{aligned}
S f_{n} & =S f-S f_{l} \\
& =\left(E_{1}^{0}+\hat{v}_{2} B^{0}\right) \partial_{v_{1}} f_{n}-\hat{v}_{1} B^{0} \partial_{v_{2}} f_{n} \\
& +\left(E_{1, n}+\hat{v}_{2} B_{n}\right) \partial_{v_{1}} f^{0}+\left(E_{2, n}-\hat{v}_{1} B_{n}\right) \partial_{v_{2}} f^{0} \\
& +\left(E_{1}+\hat{v}_{2} B\right) \partial_{v_{1}} f+\left(E_{2}-\hat{v}_{1} B\right) \partial_{v_{2}} f .
\end{aligned}
$$

We shall estimate $\left|E_{1}\right|_{1},\left|E_{2}\right|_{1},|B|_{1}$ using the field representation formulae given above for derivatives and the following duality lemma that was proved in [12]. 
Lemma 3.4. (i) If $g(x) \in L_{p e r}^{1}(0, P), \int_{0}^{P} g(x) d x=0$ and $g_{x} \in L^{1}(0, P)$, then

$$
\|g\|_{1} \leq 2 \sup _{\substack{a \in W_{p e r}^{1, \infty}(0, P) \\\left\|a_{x}\right\|_{\infty} \leq 1}} \int_{0}^{P} g_{x} a d x .
$$

(ii) If $g(x) \in L_{\text {per }}^{\infty}(0, P), \int_{0}^{P} g(x) d x=0$ and $g_{x} \in L^{\infty}(0, P)$, then

$$
\|g\|_{\infty} \leq 2 \sup _{\substack{a \in W_{p e r}^{1,1}(0, P) \\\left\|a_{x}\right\|_{1} \leq 1}} \int_{0}^{P} g_{x} a d x .
$$

Denoting by $\mathscr{L}^{*}$ the adjoint operator of $\mathscr{L}$, we have

$$
\mathscr{L}^{*}\left(\begin{array}{c}
f \\
E_{1} \\
E_{2} \\
B
\end{array}\right)=\left(\begin{array}{c}
D f+\hat{v}_{1} E_{1}+\hat{v}_{2} E_{2} \\
\int \hat{v}_{1} \mu_{e} f d v \\
\int \hat{v}_{2} \mu_{e} f d v+\partial_{x} B \\
-\int \hat{v}_{1} \mu_{p} f d v+\partial_{x} E_{2}
\end{array}\right) .
$$

\subsection{Semigroup estimate}

By $\mathbb{R}_{P}$ we mean the circle parameterized by $0 \leq x<P$. By $L^{1}$ we mean $L^{1}=$ $L^{1}\left(\mathbb{R}_{P} \times \mathbb{R}^{2}\right) \times\left(L^{1}\left(\mathbb{R}_{P}\right)^{3}\right)$ and by $L^{\infty}$ we mean $L^{\infty}=L^{\infty}\left(\mathbb{R}_{P} \times \mathbb{R}^{2}\right) \times\left(L^{\infty}\left(\mathbb{R}_{P}\right)\right)^{3}$. As in [9] we have the following lemma due to the separation of the Vlasov and Maxwell characteristics.

Lemma 3.5. If $\sup _{x} \int_{\mathbb{R}^{2}}\langle v\rangle\left(\left|\mu_{e}\right|+\left|\mu_{p}\right|\right) d v<\infty$, then $K e^{-t A} K$ is a compact operator on $L^{1}$ for all $t>0$.

Proof. The proof is a slight modification of that in [9]. Denote $w(t)=e^{-t A} K w^{\#}$ where $w^{\#}=\left[f^{\#}, E_{1}^{\#}, E_{2}^{\#}, B^{\#}\right]$ and $w(t)=\left[\delta f(t), \delta E_{1}(t), \delta E_{2}(t), \delta B(t)\right]$. Then $w(t)$ solves

$$
\begin{gathered}
\left(\partial_{t}+\hat{v}_{1} \partial_{x}-\left(E_{1}^{0}+\hat{v}_{2} B^{0}\right) \partial_{v_{1}}+\hat{v}_{1} B^{0} \partial_{v_{2}}\right) \delta f=0, \\
\partial_{t} \delta E_{1}=\int \hat{v}_{1} \delta f d v, \partial_{t} \delta B=-\partial_{x} \delta E_{2}, \\
\partial_{t} \delta E_{2}=-\partial_{x} \delta B+\int \hat{v}_{2} \delta f d v
\end{gathered}
$$

with initial conditions

$$
\begin{gathered}
\delta f(0)=\hat{v}_{1} \mu_{e} \delta E_{1}+\hat{v}_{2} \mu_{e} \delta E_{2}-\hat{v}_{1} \mu_{p} \delta B, \\
\delta E_{1}(0)=\delta E_{2}(0)=\delta B(0)=0 .
\end{gathered}
$$


As usual, we have

$$
\begin{gathered}
\delta E_{2}=\int_{0}^{t}\left[j_{2}(\tau, x-t+\tau)+j_{2}(\tau, x+t-\tau)\right] d \tau \\
\delta B=\int_{0}^{t}\left[j_{2}(\tau, x-t+\tau)-j_{2}(\tau, x+t-\tau)\right] d \tau .
\end{gathered}
$$

By (3.13) we write

$$
\delta f(t)=\delta f(0, X(0 ; t, x, v), V(0 ; t, x, v))
$$

so that

$$
j_{2}(t, x)=\int \hat{v}_{2} \delta f(t) d v=\int \hat{v}_{2} \delta f(0, X(0 ; t, x, v), V(0 ; t, x, v)) d v .
$$
is

We also use the operator splitting idea to handle $\partial_{x} E_{2}$ and $\partial_{x} B$. A typical term

$$
\begin{aligned}
I^{l}(t, x) & =\int_{0}^{t} \partial_{x} \int \hat{v}_{2} \delta f(\tau, x-t+\tau) d v d \tau \\
& =\int_{0}^{t} \int \frac{\hat{v}_{2}}{1-\hat{v}_{1}}\left(T^{+}-S\right) \delta f(\tau, x-t+\tau) d v d \tau \\
& =\int \frac{\hat{v}_{2}}{1-\hat{v}_{1}}(\delta f(t, x, v)-\delta f(0, x-\tau, v)) d v-\int_{0}^{t} \int \frac{\hat{v}_{2}}{1-\hat{v}_{1}} S \delta f(\tau, x-t+\tau) d v d \tau \\
& =I_{1}^{l}+I_{2}^{l} .
\end{aligned}
$$

Since $\left|\frac{\hat{v}_{2}}{1-\hat{v}_{1}}\right| \leq\langle v\rangle$ and there exist $c_{0}, c_{1}>0$ such that for all $t \in \mathbb{R}$

$$
c_{0}\langle V(t)\rangle \leq\langle v\rangle \leq c_{1}\langle V(t)\rangle
$$

as is easy to see, we have

$$
\begin{aligned}
\int\left|I_{1}^{l}(t, x)\right| d x & \leq C \iint\langle v\rangle|\delta f|(0) d x d v \\
& \leq C\left(\left\|E^{\#}\right\|_{1}+\left\|B^{\#}\right\|_{1}\right) .
\end{aligned}
$$

Since

$$
S \delta f=\left(\left(E_{1}^{0}+\hat{v}_{2} B^{0}\right) \partial_{v_{1}}-\hat{v}_{1} B^{0} \partial_{v_{2}}\right) \delta f=\nabla_{v} \cdot\left(\left(E_{1}^{0}+\hat{v}_{2} B^{0}\right) \delta f,-\hat{v}_{1} B^{0} \delta f\right),
$$

we have

$$
I_{2}^{l}=\int_{0}^{t} \int \nabla v\left(\frac{\hat{v}_{2}}{1-\hat{v}_{1}}\right) \cdot\left(\left(E_{1}^{0}+\hat{v}_{2} B^{0}\right) \delta f,-\hat{v}_{1} B^{0} \delta f\right)(\tau, x-t+\tau) d v d \tau
$$


Using the inequality $\left|\nabla_{v}\left(\frac{\hat{v}_{2}}{1-\hat{v}_{1}}\right)\right| \leq C\langle v\rangle$ as well as (3.14), (3.15), we have

$$
\begin{aligned}
& \int\left|I_{2}^{l}(t, x)\right| d x \\
& \leq C \int_{0}^{t} \int_{\mathbb{R}_{P} \times \mathbb{R}^{2}}\langle V(t-s ; t, x, v)\rangle|\delta f|(0, X(t-s ; t, x, v) V(t-s ; t, x, v)) d x d v d s \\
& =C t \int_{\mathbb{R}_{P} \times \mathbb{R}^{2}}\langle v\rangle|\delta f|(0) d x d v \leq C t\left(\left\|E^{\#}\right\|_{1}+\left\|B^{\#}\right\|_{1} .\right)
\end{aligned}
$$

Here we used $C$ to denote a general constant and we used the fact that the Jacobian of the mapping $x, v \rightarrow(X(t-s ; t, x, v) V(t-s ; t, x, v))$ is 1 . Since

$$
\partial_{x} \delta E_{1}(t)+\int \delta f(t) d v=\partial_{x} \delta E_{1}(0)+\int \delta f(0) d v=0,
$$

we have

$$
\left\|\partial_{x} \delta E_{1}(t)\right\|_{1} \leq\|\delta f(t)\|_{1}=\|\delta f(0)\|_{1} \leq C\left(\left\|E^{\#}\right\|_{1}+\left\|B^{\#}\right\|_{1}\right) .
$$

Combining the preceding estimates, we have

$$
\left\|\partial_{x} \delta E\right\|_{1}+\left\|\partial_{x} \delta B\right\|_{1} \leq\left(C_{1} t+C_{2}\right)\left(\left\|E^{\#}\right\|_{1}+\left\|B^{\#}\right\|_{1}\right) .
$$

Now

$$
\partial_{x} K w(t)=\left(\begin{array}{c}
\hat{v}_{1} \mu_{e} \delta E_{1}(t)+\hat{v}_{2} \mu_{e} \delta E_{2}(t)-\hat{v}_{1} \mu_{p} \delta B(t) \\
0 \\
0 \\
0
\end{array}\right)
$$

so that

$$
\left\|\partial_{x} K w(t)\right\|_{1} \leq C\left(\left\|\partial_{x} \delta E(t)\right\|_{1}+\left\|\partial_{x} \delta B(t)\right\|_{1}\right) \leq C\left(C_{1} t+C_{2}\right)\left(\left\|E^{\#}\right\|_{1}+\left\|B^{\#}\right\|_{1}\right) .
$$

Therefore $w^{\#} \rightarrow K e^{-t A} K w^{\#}$ is a compact linear operator.

The preceding lemma implies that $K$ is $A$-smoothing. Combining this fact with the following functional analysis lemma used in [9], we have control of the growth of the linear semigroup.

Lemma 3.6. For all $\delta>0$, the spectrum of $\mathscr{L}$ in $\{\operatorname{Re} \lambda>\delta\}$ consists of a finite number of eigenvalues of finite multiplicity. If $\lambda_{1}$ denotes the eigenvalue of $\mathscr{L}$ with maximal real part, and $\Lambda>\max \left\{0, \operatorname{Re} \lambda_{1}\right\}$, then there exists $C_{\Lambda}>0$ such that

$$
\left\|e^{t \mathscr{L}}\right\|_{L^{1} \rightarrow L^{1}} \leq C_{\Lambda} e^{\Lambda t} .
$$

We now define the two spaces

$$
L^{1, k}=\left\{f(x, v)\left|\|f\|=\int_{\mathbb{R}_{P} \times \mathbb{R}^{2}}\langle v\rangle^{k}\right| f \mid d x d v<\infty\right\}
$$

and

$$
L^{\infty, k}=\left\{f(x, v) \mid\|f\|=\sup \frac{|f|}{\langle v\rangle^{k}}<\infty\right\} .
$$


Using the notation of the previous lemma, we have

Lemma 3.7. Assume $\sup _{x} \int_{\mathbb{R}^{2}}\langle v\rangle^{k}\left(\left|\mu_{e}\right|+\left|\mu_{p}\right|\right) d v<\infty$. Let $f_{0} \in L^{1, k}\left(\mathbb{R}_{P} \times \mathbb{R}^{2}\right)$ and let $\left(f(t), E_{1}(t), E_{2}(t), B(t)\right)=e^{t \mathscr{L}}\left(f_{0}, 0,0,0\right)$. If $\lambda_{1}$ denotes the eigenvalue with maximal real part and $\Lambda>\max \left\{0, \operatorname{Re} \lambda_{1}\right\}$, then there exists $C_{\Lambda}>0$ such that

$$
\|f(t)\|_{L^{1, k}} \leq C_{\Lambda} e^{\Lambda t}\left\|f_{0}\right\|_{L^{1, k}} .
$$

Proof. We prove it by deduction. The case $k=0$ follows from Lemma 3.6. Moreover, there exists $C_{\Lambda}^{\prime}>0$ such that

$$
\left(\left\|E_{1}\right\|_{1}+\left\|E_{2}\right\|_{1}+\|B\|_{1}\right)(t) \leq C_{\Lambda}^{\prime} e^{\Lambda t}\left\|f_{0}\right\|_{L^{1}}
$$

Suppose the conclusion is true for $k-1$; that is, there exists $C_{\Lambda}^{\prime \prime}>0$ such that $\|f(t)\|_{L^{1, k-1}} \leq C_{\Lambda}^{\prime \prime} e^{\Lambda t}\left\|f_{0}\right\|_{L^{1, k-1}}$. We multiply (3.2) by $\langle v\rangle^{k}$ and rewrite it as

$$
\begin{aligned}
& \partial_{t}\left(\langle v\rangle^{k} f\right)+\hat{v}_{1} \partial_{x}\left(\langle v\rangle^{k} f\right)-\left(E_{1}^{0}+\hat{v}_{2} B^{0}\right) \partial_{v_{1}}\left(\langle v\rangle^{k} f\right)+\hat{v}_{1} B^{0} \partial_{v_{2}}\langle v\rangle^{k} f \\
& =\left(E_{1}+\hat{v}_{2} B\right)\langle v\rangle^{k} f \partial_{v_{1}} f^{0}+\left(E_{2}-\hat{v}_{1} B\right)\langle v\rangle^{k} f \partial_{v_{2}} f \\
& -\left(E_{1}^{0}+\hat{v}_{2} B^{0}\right) k\langle v\rangle^{k-1} \hat{v}_{1} f+\hat{v}_{1} B^{0} k\langle v\rangle^{k-1} \hat{v}_{2} f .
\end{aligned}
$$

Integrating it along a trajectory, we have

$$
\begin{aligned}
\langle v\rangle^{k} f(t) & =\langle V(0 ; t, x, v)\rangle^{k} f_{0}(X(0 ; t, x, v), V(0 ; t, x, v)) \\
+ & \int_{0}^{t}\left\{\left(E_{1}+\hat{v}_{2} B\right)\langle v\rangle^{k} \partial_{v_{1}} f_{0}+\left(E_{2}-\hat{v}_{1} B\right)\langle v\rangle^{k} \partial_{v_{2}} f_{0}\right. \\
- & \left(E_{1}^{0}+\hat{v}_{2} B^{0}\right) k\langle v\rangle^{k-1} \hat{v}_{1} f+\hat{v}_{1} B^{0} k\langle v\rangle^{k-1} \hat{v}_{2} f \\
& (t-s, X(t-s ; t, x, v), V(t-s ; t, x, v))\} d s
\end{aligned}
$$

Taking absolute values and integrating, we have

$$
\begin{aligned}
\|f(t)\|_{L^{1, k}} & \leq\left\|f_{0}\right\|_{L^{1, k}}+C \int_{0}^{t}\left(\left\|E_{1}\right\|_{1}+\left\|E_{2}\right\|_{1}+\|B\|_{1}+\|f\|_{L^{1, k-1}}\right)(t-s) d s \\
& \leq\left\|f_{0}\right\|_{L^{1, k}}+C \int_{0}^{t}\left(C_{\Lambda}^{\prime}+C_{\Lambda}^{\prime \prime}\right) e^{\Lambda(t-s)} d s \\
& \leq C_{\Lambda} e^{\Lambda t}
\end{aligned}
$$

for some $C_{\Lambda}>0$, where we have again used the fact that the Jacobian of the mapping $(x, v) \rightarrow(X(s ; t, x, v), V(s ; t, x, v))$ is unity.

The following estimate of the dual semigroup will be used later.

Lemma 3.8. Assume $\sup _{x} \int_{\mathbb{R}^{2}}\langle v\rangle\left(\left|\mu_{e}\right|+\left|\mu_{p}\right|\right) d v<\infty$. If $\lambda_{1}$ denotes an eigenvalue of $\mathscr{L}$ with maximal real part, and $\Lambda>\max \left\{0, \operatorname{Re} \lambda_{1}\right\}$, then there exists $C_{\Lambda}>0$ such that

(I)

$$
\left\|e^{t \mathscr{L}^{*}}\right\|_{L^{\infty} \rightarrow L^{\infty}} \leq C_{\Lambda} e^{\Lambda t}
$$


(II) Assume $\sup _{x} \int_{\mathbb{R}^{2}}\langle v\rangle^{k}\left(\left|\mu_{e}\right|+\left|\mu_{p}\right|\right) d v<\infty$. If $f_{0} \in L^{\infty, k}\left(\mathbb{R}_{P} \times \mathbb{R}^{2}\right)$ and

$$
\left(f(t), E_{1}(t), E_{2}(t), B(t)\right)=\exp \left(t \mathscr{L}^{*}\right)\left(f_{0}, 0,0,0\right)
$$

then

$$
\|f(t)\|_{L^{\infty, k}} \leq C_{\Lambda} e^{\Lambda t}\left\|f_{0}\right\|_{L^{\infty, k}}
$$

(III) There exists $C_{1}, C_{2}>0$ such that if

$$
w(0)=\left(f(0), E_{1}(0), E_{2}(0), B(0)\right) \in W^{1, \infty}\left(\mathbb{R}_{P} \times \mathbb{R}^{2}\right) \times\left(W^{1, \infty}\left(\mathbb{R}_{P}\right)\right)^{3}
$$

and

$$
\left(f(t), E_{1}(t), E_{2}(t), B(t)\right)=\exp \left(t \mathscr{L}^{*}\right)\left(f(0), E_{1}(0), E_{2}(0), B(0)\right),
$$

then

$$
\left|\partial_{v} f(t)\right| \leq C\left(|J(t)|_{2}+C_{\Lambda} \int_{0}^{t} e^{\Lambda(t-s)}|J(s)|_{2} d s\right)\|w(0)\|_{W^{1, \infty}}
$$

(IV) Assume sup $\int_{\mathbb{R}^{2}}\langle v\rangle^{k+1}\left(\left|\mu_{e}\right|+\left|\mu_{p}\right|\right) d v<\infty$. Let $f_{0} \in L^{\infty, k}\left(\mathbb{R}_{P} \times \mathbb{R}^{2}\right), \partial_{v} f_{0} \in$ $L^{\infty, k^{\prime}}\left(\mathbb{R}_{P} \times \mathbb{R}^{2}\right)$ and

$$
\left(f(t), E_{1}(t), E_{2}(t), B(t)\right)=\exp \left(t \mathscr{L}^{*}\right)\left(f_{0}, 0,0,0\right),
$$

then

$$
\left|\partial_{v} f(t)\right| \leq C\left\|\partial_{v} f_{0}\right\|_{L^{\infty, k^{\prime}}}\langle v\rangle^{k^{\prime}} J(t)+C_{\Lambda}\left\|f_{0}\right\|_{L^{\infty, k}} \int_{0}^{t} e^{\Lambda(t-s)}|J(s)|_{2} d s .
$$

Proof. Conclusions (I) and (II) are immediate since they are the dual versions of Lemmas 3.6 and 3.7 respectively, noting that

$$
\|f\|_{L^{\infty, k}}=\sup _{\|g\|_{L^{1, k}} \leq 1} \int f g d x d v .
$$

We will now prove (III). By definition, $\left(f(t), E_{1}(t), E_{2}(t), B(t)\right)$ is the solution of the system

$$
\begin{gathered}
\partial_{t} f=\left(\hat{v}_{1} \partial_{x}-\left(E_{1}^{0}+\hat{v}_{2} B^{0}\right) \partial_{v_{1}}+\hat{v}_{1} B^{0} \partial_{v_{2}}\right) f+\hat{v}_{1} E_{1}+\hat{v}_{2} E_{2} \\
\partial_{t} E_{1}=\int \hat{v}_{1} \mu_{e} f d v \\
\partial_{t} E_{2}=\int \hat{v}_{2} \mu_{e} f d v+\partial_{x} B \\
\partial_{t} B=-\int \hat{v}_{1} \mu_{p} f d v+\partial_{x} E_{2} .
\end{gathered}
$$


By (3.19) and (3.20) we have the formulae

$$
\begin{aligned}
E_{2}(t, x) & =\frac{1}{2}\left[E_{2}(0, x-t)+E_{2}(0, x+t)+B(0, x-t)+B(0, x+t)\right] \\
& +\frac{1}{2} \int_{0}^{t} \int\left(\hat{v}_{2} \mu_{e}-\hat{v}_{1} \mu_{p}\right) f d v(\tau, x+t-\tau) d \tau \\
& +\frac{1}{2} \int_{0}^{t} \int\left(\hat{v}_{2} \mu_{e}+\hat{v}_{1} \mu_{p}\right) f d v(\tau, x-t+\tau) d \tau
\end{aligned}
$$

and

$$
\begin{aligned}
B(t, x) & =\frac{1}{2}\left[E_{2}(0, x-t)-E_{2}(0, x+t)+B(0, x-t)+B(0, x+t)\right] \\
& +\frac{1}{2} \int_{0}^{t} \int\left(\hat{v}_{2} \mu_{e}-\hat{v}_{1} \mu_{p}\right) f d v(\tau, x+t-\tau) d \tau \\
& -\frac{1}{2} \int_{0}^{t} \int\left(\hat{v}_{2} \mu_{e}+\hat{v}_{1} \mu_{p}\right) f d v(\tau, x-t+\tau) d \tau
\end{aligned}
$$

We use the operator splitting idea as before to deal with $\partial_{x} E_{2}$ and $\partial_{x} B$. A typical term is

$$
\begin{aligned}
I^{d}(t, x) & =\int_{0}^{t} \int\left(\hat{v}_{2} \partial_{x}\left(\mu_{e} f\right)-\hat{v}_{1} \partial_{x}\left(\left(\mu_{p} f\right)\right)\right)(\tau, x-t+\tau) d v d \tau \\
& =\int_{0}^{t} \int\left(\frac{\hat{v}_{2}}{1-\hat{v}_{1}}\left(T^{+}-S^{-}\right) \mu_{e} f-\frac{\hat{v}_{1}}{1-\hat{v}_{1}}\left(T^{+}-S^{-}\right) \mu_{p} f\right)(\tau, x-t+\tau) d v d \tau
\end{aligned}
$$

where $T^{+}=\partial_{t}+\partial_{x}$ and $S^{-}=\partial_{t}-\hat{v}_{1} \partial_{x}$. We estimate each term above as follows.

$$
\begin{aligned}
\left|I_{1}^{d}(t, x)\right| & =\left|\int_{0}^{t} \int \frac{\hat{v}_{2}}{1-\hat{v}_{1}} T^{+}\left(\mu_{e} f\right)(\tau, x-t+\tau) d v d \tau\right| \\
& =\left|\int \frac{\hat{v}_{2}}{1-\hat{v}_{1}}\left(\mu_{e} f(t, x, v)-\mu_{e} f(0, x-t, v)\right) d v\right| \\
& \leq C \sup _{x} \int\langle v\rangle\left|\mu_{e}\right| d v\left(\|f(t)\|_{\infty}+\left\|f_{0}\right\|_{\infty}\right) \\
& \leq C_{\Lambda}^{\prime} e^{\Lambda t}\|w(0)\|_{\infty}
\end{aligned}
$$

by (I). We have

$$
\begin{aligned}
& I_{2}^{d}(t, x) \\
= & \int_{0}^{t} \int \frac{\hat{v}_{2}}{1-\hat{v}_{1}} S^{-}\left(\mu_{e} f\right)(\tau, x-t+\tau) d v d \tau \\
= & \int_{0}^{t} \int \frac{\hat{v}_{2}}{1-\hat{v}_{1}}\left(\left(-\left(E_{1}^{0}+\hat{v}_{2} B^{0}\right) \partial_{v_{1}}+\hat{v}_{1} B^{0} \partial_{v_{2}}\right) \mu_{e} f+\hat{v}_{1} \mu_{e} E_{1}+\hat{v}_{2} \mu_{e} E_{2}\right) d v(\tau, x-t+\tau) d \tau \\
= & \int_{0}^{t} \int\left(\nabla v\left(\frac{\hat{v}_{2}}{1-\hat{v}_{1}}\right) \cdot\left(E_{1}^{0}+\hat{v}_{2} B^{0}, \hat{v}_{1} B^{0}\right) \mu_{e} f+\frac{\hat{v}_{2}}{1-\hat{v}_{1}}\left(\hat{v}_{1} \mu_{e} E_{1}+\hat{v}_{2} \mu_{e} E_{2}\right)\right) d v(\tau, x-t+\tau) d \tau
\end{aligned}
$$


since by (3.17)

(3.24) $\partial_{t}\left(\mu_{e} f\right)=\left(\hat{v}_{1} \partial_{x}-\left(E_{1}^{0}+\hat{v}_{2} B^{0}\right) \partial_{v_{1}}+\hat{v}_{1} B^{0} \partial_{v_{2}}\right)\left(\mu_{e} f\right)+\hat{v}_{1} \mu_{e} E_{1}+\hat{v}_{2} \mu_{e} E_{2}$.

Thus

$$
\begin{aligned}
\left|I_{2}^{d}(t, x)\right| & \leq C \sup _{x} \int\langle v\rangle\left|\mu_{e}\right| d v \int_{0}^{t}\left(\|f\|_{\infty}+\left\|E_{1}\right\|_{\infty}+\left\|E_{2}\right\|_{\infty}\right)(\tau) d \tau \\
& \leq C_{\Lambda}^{\prime \prime} e^{\Lambda t}\|w(0)\|_{\infty} .
\end{aligned}
$$

The other two terms in (3.23) can be estimated in the same way and we finally get $\left|I^{d}(t, x)\right| \leq C_{\Lambda} e^{\Lambda t}\|w(0)\|_{\infty}$. Thus

$$
\left(\left|\partial_{x} E_{2}\right|+\left|\partial_{x} B\right|\right)(t) \leq C_{\Lambda} e^{\Lambda t}\|w(0)\|_{W^{1, \infty}} .
$$

Integrating (3.24) with respect to $v$, we have

$$
\partial_{t} \partial_{x} E_{1}=\partial_{x} \int \hat{v}_{1} \mu_{e} f d v=\partial_{t} \int \mu_{e} f d v-\int \hat{v}_{2} \mu_{e} d v E_{2}
$$

So

$$
\partial_{x} E_{1}(t, x)=\partial_{x} E_{1}(0)+\int \mu_{e} f(t) d v-\int \mu_{e} f(0) d v-\int \hat{v}_{2} \mu_{e} d v \int_{0}^{t} E_{2}(s) d s,
$$

which combined with (I) implies that

$$
\left|\partial_{x} E_{1}\right|(t) \leq C_{\Lambda} e^{\Lambda t}\|w(0)\|_{W^{1, \infty}} .
$$

Now integrating (3.17) along a trajectory, we have

$$
\begin{aligned}
f(t, x, v) & =f(0, X(t ; x, v), V(t ; x, v)) \\
& +\int_{0}^{t}\left(\hat{V}_{1}(s) E_{1}(t-s, X(s))+\hat{V}_{2}(s) E_{2}(t-s, X(s))\right) d s
\end{aligned}
$$

so

$$
\begin{aligned}
\left|\partial_{v} f(t)\right| & \leq\left|\partial_{v} f(0)\right|_{\infty}|J(t)|_{2} \\
& +C \int_{0}^{t}\left(\left|E_{1}\right|_{\infty}+\left|E_{2}\right|_{\infty}+\left|\partial_{x} E_{1}\right|_{\infty}+\left|\partial_{x} E_{2}\right|_{\infty}\right)(t-s)|J(s)|_{2} d s,
\end{aligned}
$$

which implies (3.16) by (I), (3.25) and (3.27).

Now we prove (IV). We use $C_{\Lambda}$ to denote some general constant only depending on $\Lambda$. By (II), (3.15) and (3.18),

$$
\begin{aligned}
\left|E_{1}(t, x)\right| & \leq \int_{0}^{t} \int\left|\hat{v}_{1} \mu_{e} f(s) d v\right| d s \leq C \sup _{x} \int_{\mathbf{R}^{2}}\langle v\rangle^{k}\left|\mu_{e}\right| d v \int_{0}^{t}\|f(s)\|_{\mathbf{L}^{\infty, k}} d s \\
& \leq C_{\Lambda}\left\|f_{0}\right\|_{\mathbf{L}^{\infty, k}} e^{\Lambda t} .
\end{aligned}
$$

Similarly by (3.21), (3.22) and (3.26),

$$
\left|E_{2}(t, x)\right|+|B(t, x)|+\left|\partial_{x} E_{1}(t, x)\right| \leq C_{\Lambda}\left\|f_{0}\right\|_{L^{\infty, k},} e^{\Lambda t} .
$$


For $\partial_{x} E_{2}$ and $\partial_{x} B$, we use the same operator splitting procedure as in the proof of (III). It is easy to see that

$$
\begin{aligned}
\left|I_{1}^{d}(t, x)\right| & \leq C \sup _{x} \int\langle v\rangle^{k+1}\left|\mu_{e}\right| d v\left(\|f(t)\|_{L^{\infty, k}}+\left\|f_{0}\right\|_{L^{\infty, k}}\right) \\
& \leq C_{\Lambda}\left\|f_{0}\right\|_{L^{\infty, k}} e^{\Lambda t}
\end{aligned}
$$

and

$$
\begin{aligned}
\left|I_{2}^{d}(t, x)\right| & \leq C \sup _{x} \int\langle v\rangle^{k+1}\left|\mu_{e}\right| d v \int_{0}^{t}\left(\|f(\tau)\|_{L^{\infty, k}}+\left\|E_{1}\right\|_{\infty}+\left\|E_{2}\right\|_{\infty}\right)(\tau) d \tau \\
& \leq C_{\Lambda}\left\|f_{0}\right\|_{L^{\infty, k}} e^{\Lambda t}
\end{aligned}
$$

by (II), (3.30) and (3.31). Thus

$$
\left(\left|\partial_{x} E_{2}\right|+\left|\partial_{x} B\right|\right)(t) \leq C_{\Lambda} e^{\Lambda t}\left\|f_{0}\right\|_{L^{\infty, k}}
$$

So from (3.29) we have

$$
\begin{aligned}
\left|\partial_{v} f(t)\right| & \leq\langle V(t)\rangle^{k^{\prime}}\left|\partial_{v} f_{0}\right|_{L^{\infty, k^{\prime}}}|J(t)|_{2} \\
& +C \int_{0}^{t}\left(\left|E_{1}\right|_{\infty}+\left|E_{2}\right|_{\infty}+\left|\partial_{x} E_{1}\right|_{\infty}+\left|\partial_{x} E_{2}\right|_{\infty}\right)(t-s)|J(s)|_{2} d s \\
& \leq C\langle v\rangle^{k^{\prime}}\left|\partial_{v} f_{0}\right|_{L^{\infty, k^{\prime}}}|J(t)|_{2}+C_{\Lambda}\left\|f_{0}\right\|_{L^{\infty, k}} \int_{0}^{t} e^{\Lambda(t-s)}|J(s)|_{2} d s
\end{aligned}
$$

by (3.15), (3.30), (3.31) and (3.32).

\subsection{Bootstrap estimate}

Lemma 3.9. Let $w^{0}=\left[f^{0}=\mu(e, p), E_{1}^{0}(x), 0, B^{0}(x)\right]$ be a steady state such that $\sup _{x} \int_{\mathbb{R}^{2}}\langle v\rangle^{2}\left(\left|\mu_{e}\right|+\left|\mu_{p}\right|\right) d v<\infty$. Let $w^{\delta}=\left[\bar{f}^{\delta}, \bar{E}_{1}^{\delta}, \bar{E}_{2}^{\delta}, \bar{B}^{\delta}\right]$ be a $W^{1, \infty}$ solution with $\langle v\rangle^{2} \bar{f}^{\delta} \in L^{1}$ to the (1 $\left.\frac{1}{2}\right) R V M$ system (3.1d) of period $P$, which satisfies the conditions

$$
\left\|\langle v\rangle^{2}\left(\bar{f}^{\delta}(0)-f^{0}\right)\right\|_{1}+\left\|\bar{E}_{1}^{\delta}(0)-E_{1}^{0}\right\|_{1}+\left\|\bar{E}_{2}^{\delta}(0)\right\|_{1}+\left\|\bar{B}^{\delta}(0)-B^{0}\right\|_{1} \leq \delta
$$

and

$$
\left\|\bar{E}_{1}^{\delta}(t)-E_{1}^{0}\right\|_{1}+\left\|\bar{E}_{2}^{\delta}(t)\right\|_{1}+\left\|\bar{B}^{\delta}(t)-B^{0}\right\|_{1} \leq C_{0} \delta e^{\omega t}
$$

with $\omega>0$ and $t \leq T$. Then there exist $D>0$ and $\theta>0$, depending on $C_{0}$ and $\omega$, such that

$$
\left\|\langle v\rangle^{2}\left(\bar{f}^{\delta}(t)-f^{0}\right)\right\|_{1}+\left\|w^{\delta}(t)-w^{0}\right\|_{\infty} \leq D \delta e^{\omega t},
$$

for $t$ in the interval $\left[0, \min \left(T, \frac{1}{\omega} \ln \frac{\theta}{\delta}\right)\right]$. 
Proof. Denote $w(t)=w^{\delta}(t)-w^{0}=\left[f(t), E_{1}(t), E_{2}(t), B(t)\right]$.

Step 1: Estimate of $\left\|E_{1}(t)\right\|_{\infty}+\left\|E_{2}(t)\right\|_{\infty}+\|B(t)\|_{\infty}$.

The proof is almost the same as in [9]. We note that (3.1a) can be rewritten as

$$
\begin{aligned}
& \left(\partial_{t}+\hat{v}_{1} \partial_{x}-\left(\bar{E}_{1}^{\delta}+\hat{v}_{2} \bar{B}^{\delta}\right) \partial_{\nu_{1}}-\left(\bar{E}_{2}^{\delta}-\hat{v}_{1} \bar{B}^{\delta}\right) \partial_{v_{2}}\right) f \\
& =\left(E_{1}+\hat{v}_{2} B\right) \partial_{v_{1}} f^{0}+\left(E_{2}-\hat{v}_{1} B\right) \partial_{v_{2}} f^{0}
\end{aligned}
$$

and thus

$$
\begin{aligned}
& \left(\partial_{t}+\hat{v}_{1} \partial_{x}-\left(\bar{E}_{1}^{\delta}+\hat{v}_{2} \bar{B}^{\delta}\right) \partial_{v_{1}}-\left(\bar{E}_{2}^{\delta}-\hat{v}_{1} \bar{B}^{\delta}\right) \partial_{v_{2}}\right)(\langle v\rangle|f|) \\
& =\left(\left(E_{1}+\hat{v}_{2} B\right)\langle v\rangle \partial_{v_{1}} f^{0}+\left(E_{2}-\hat{v}_{1} B\right)\langle v\rangle \partial_{v_{2}} f^{0}\right) \operatorname{sgn} f \\
& \quad-\left(\bar{E}_{1}^{\delta}+\hat{v}_{2} \bar{B}^{\delta}\right) \hat{v}_{1}|f|-\left(\bar{E}_{2}^{\delta}-\hat{v}_{1} \bar{B}^{\delta}\right) \hat{v}_{2}|f| .
\end{aligned}
$$

Using the above equation and following the proof in [9], we get the desired bootstrap estimate: there exist $D_{1}>0$ and $\theta>0$, such that

$$
\left\|E_{1}(t)\right\|_{\infty}+\left\|E_{2}(t)\right\|_{\infty}+\|B(t)\|_{\infty} \leq D_{1} \delta e^{\omega t},
$$

for $t$ in the interval $\left[0, \min \left(T, \frac{1}{\omega} \ln \frac{\theta}{\delta}\right)\right]$.

Step 2: Estimate of $\|f(t)\|_{\infty}$.

Denote by $\left(X^{*}(s ; t, x, v), V^{*}(s ; t, x, v)\right)$ the perturbed trajectory satisfying

$$
\left\{\begin{array}{c}
\dot{X}^{*}=\hat{V}_{1}^{*} \\
\dot{V}_{1}^{*}=-\left(\bar{E}_{1}^{\delta}\left(t, X^{*}\right)+\hat{V}_{2}^{*} \bar{B}^{\delta}\left(t, X^{*}\right)\right) \\
\dot{V}_{2}^{*}=-\left(\bar{E}_{2}^{\delta}\left(t, X^{*}\right)-\hat{V}_{1}^{*} \bar{B}^{\delta}\left(t, X^{*}\right)\right) .
\end{array}\right.
$$

with $\left(X^{*}(t), V^{*}(t)\right)=(x, v)$. Then

$$
\begin{aligned}
\bar{f}^{\delta}(t, x, v) & =\bar{f}^{\delta}\left(0, X^{*}(0), X^{*}(0)\right) \\
& =\mu(\tilde{e}(0), \tilde{p}(0))+f\left(0, X^{*}(0), V^{*}(0)\right),
\end{aligned}
$$

where

$$
\tilde{e}(s)=\sqrt{1+V_{1}^{*}(s)^{2}+V_{2}^{*}(s)^{2}}-\phi^{0}\left(X^{*}(s)\right), \tilde{p}(s)=V_{2}^{*}(s)+\psi^{0}\left(X^{*}(s)\right) .
$$

We have

$$
\begin{aligned}
|\tilde{e}(0)-e| & =\left|\int_{0}^{t} \frac{d}{d s} \tilde{e}(s) d s\right|=\left|\int_{0}^{t}\left(-E_{2}\left(s, X^{*}(s)\right) \hat{V}_{2}^{*}(s)+E_{1}\left(s, X^{*}\right) \hat{V}_{1}^{*}(s)\right)\right| d s \\
& \leq \int_{0}^{t}\left(\left|E_{1}(s)\right|_{\infty}+\left|E_{2}(s)\right|_{\infty}\right) d s \leq \frac{D}{\omega} \delta e^{\omega t}
\end{aligned}
$$

and similarly $|\tilde{p}(0)-e| \leq \frac{D}{\omega} \delta e^{\omega t}$. Thus

$$
|f(t)|_{\infty} \leq|\mu(\tilde{e}(0), \tilde{p}(0))-\mu(e, p)|+|f(0)|_{\infty} \leq D_{2} \delta e^{\omega t}
$$

for some $D_{2}$ depending on $D, \mu$ and $\omega$. 
Step 3: Estimate of $\left\|\langle v\rangle^{2} f(t)\right\|_{1}$.

Integrating (3.33) along a perturbed trajectory, we have

$$
\begin{aligned}
& f(t, x, v)=f\left(0, X^{*}(0), X^{*}(0)\right) \\
& \quad+\int_{0}^{t}\left(\left(E_{1}+\hat{v}_{2} B\right) \partial_{v_{1}} f^{0}+\left(E_{2}-\hat{v}_{1} B\right) \partial_{v_{2}} f^{0}\right)\left(t-s, X^{*}(t-s), V^{*}(t-s)\right) d s
\end{aligned}
$$

so that

$$
\|f(t)\|_{1} \leq\|f(0)\|_{1}+C \int_{0}^{t}\left(\left\|E_{1}\right\|_{1}+\left\|E_{2}\right\|_{1}+\|B\|_{1}\right)(s) d s \leq D_{3} \delta e^{\omega t}
$$

for some $D_{3}$ only depending on $C_{0}, \omega$, as $t \leq T$. Integrating (3.34) and using the bound in Step 1 (assuming $\theta \leq 1$ ), we have

$$
\begin{aligned}
\|\langle v\rangle f(t)\|_{1} & \leq\|\langle v\rangle f(0)\|_{1}+C \int_{0}^{t}\left(\left\|E_{1}\right\|_{1}+\left\|E_{2}\right\|_{1}+\|B\|_{1}\right)(s) d s \\
& +C \int_{0}^{t}\left(\left\|w^{0}\right\|_{\infty}+\left\|E_{1}\right\|_{\infty}(s)+\left\|E_{2}\right\|_{\infty}(s)+\|B\|_{\infty}(s)\right)\|f\|_{1}(s) d s \\
& \leq D_{4} \delta e^{\omega t}
\end{aligned}
$$

for some $D_{4}$ only depending on $D_{3}, D_{1}, \omega$. By (3.33), we have

$$
\begin{aligned}
& \left(\partial_{t}+\hat{v}_{1} \partial_{x}-\left(\bar{E}_{1}^{\delta}+\hat{v}_{2} \bar{B}^{\delta}\right) \partial_{v_{1}}-\left(\bar{E}_{2}^{\delta}-\hat{v}_{1} \bar{B}^{\delta}\right) \partial_{v_{2}}\right)\left(\langle v\rangle^{2}|f|\right) \\
& =\left(\left(E_{1}+\hat{v}_{2} B\right)\langle v\rangle^{2} \partial_{v_{1}} f^{0}+\left(E_{2}-\hat{v}_{1} B\right)\langle v\rangle^{2} \partial_{v_{2}} f^{0}\right) s g n f \\
& \quad-2\left(\bar{E}_{1}^{\delta}+\hat{v}_{2} \bar{B}^{\delta}\right) \hat{v}_{1}\langle v\rangle|f|-2\left(\bar{E}_{2}^{\delta}-\hat{v}_{1} \bar{B}^{\delta}\right) \hat{v}_{2}\langle v\rangle|f| .
\end{aligned}
$$

Integrating the preceding equations and using the estimate on $\|\langle v\rangle f(t)\|_{1}$, we deduce

$$
\left\|\langle v\rangle^{2} f(t)\right\|_{1} \leq D_{5} \delta e^{\omega t}
$$

for some $D_{5}$ depending on $D_{4}, D_{3}, D_{1}, \omega$. This completes the proof of the lemma.

\subsection{Proof of nonlinear instability}

We consider the perturbed initial data

$$
\bar{E}_{1}^{\delta}(0)=E_{1}^{0}+\delta E_{g 1}, \bar{E}_{2}^{\delta}(0)=\delta E_{g 2}, \bar{B}^{\delta}(0)=B^{0}+\delta B_{g}, \bar{f}^{\delta}(0)=f^{0}+\delta f_{g},
$$

where $\left(f_{g}, E_{g 1}, E_{g 2}, B_{g}\right)$ is the growing mode and $\delta$ is a small parameter. (In case the growing mode is not real, we take the imaginary parts of the growing mode as in [9].) By Lemma 3.3, $\bar{f}^{\delta}(0) \geq 0$ for sufficiently small $\delta$. We consider the solution to the $1 \frac{1}{2} \mathrm{D}$ RVM system (3.1d) with the initial data given above. Furthermore, as mentioned above, we have the well-posedness of the system (3.1d), which is more than sufficient for our purpose of proving nonlinear instability. 
We decompose the perturbation of the field into its linear and nonlinear parts as in Section 3.2, writing

$$
E_{1}=E_{1, l}+E_{1, n}, E_{2}=E_{2, l}+E_{2, n}, B=B_{l}+B_{n} .
$$

If we could show that for some $\theta, c_{3}>0$,

$$
\left\|E_{1, n}(t)\right\|_{1}+\left\|E_{2, n}(t)\right\|_{1}+\left\|B_{n}(t)\right\|_{1} \leq c_{3}\left(\delta e^{\omega t}\right)^{2}
$$

for $t \leq T^{*}=\frac{1}{\omega} \ln \frac{\theta}{\delta}$, then nonlinear instability would follow by a standard argument as in [9] (see also [12]), in the norm $\left\|E_{1}(t)\right\|_{1}+\left\|E_{2}(t)\right\|_{1}+\|B(t)\|_{1}$.

In order to prove (3.36), we use the field representation formulae for the derivatives and the Duality Lemma 3.4. First we estimate $\left\|E_{1, n}\right\|_{1}$. By Lemma 3.4,

$$
\left\|E_{1, n}(t)\right\|_{1} \leq\left|\int_{0}^{P} E_{1, n}(t) d x\right|+2 \sup _{\substack{a \in W_{\text {per }}^{1, \infty}(0, P) \\\left\|a_{x}\right\|_{\infty} \leq 1}} \int_{0}^{P} \partial_{x} E_{1, n}(t) a(x) d x .
$$

We have

$$
\begin{aligned}
& \int_{0}^{P} E_{1, n}(t) d x=\int_{0}^{t} \iint_{\mathbb{R}_{P} \times \mathbb{R}^{2}} \hat{v}_{1} f_{n}(s) d v d x d s \\
& =\int_{0}^{t} \int_{0}^{s}\left(\left(\begin{array}{c}
\hat{v}_{1} \\
0 \\
0 \\
0
\end{array}\right), e^{(s-u) \mathscr{L}}\left(\begin{array}{c}
-\nabla_{v} \cdot\left(\left(E_{1}+\hat{v}_{2} B\right) f,\left(E_{1}-\hat{v}_{1} B\right) f\right)(u) \\
0 \\
0 \\
0
\end{array}\right)\right) d u d s \\
& =\int_{0}^{t} \int_{0}^{s} \iint_{\mathbb{R}_{P} \times \mathbb{R}^{2}} \nabla v g_{1}(s-u, x, v) \cdot\left(\left(E_{1}+\hat{v}_{2} B\right) f,\left(E_{1}-\hat{v}_{1} B\right) f\right)(u) d x d v d u d s
\end{aligned}
$$

where $g_{1}(t, x, v)$ is the first component of $e^{t \mathscr{L}^{*}}\left[\hat{v}_{1}, 0,0,0\right]$. Since $\left|\nabla_{v}\left(\hat{v}_{1}\right)\right|+\left|\hat{v}_{1}\right| \leq$ 3 , Lemma 3.8 (III) for $\omega<\Lambda<2 \omega$ implies

$$
\left|\nabla v g_{1}(t, x, v)\right| \leq C\left(|J(t)|_{2}+C_{\Lambda} \int_{0}^{t} e^{\Lambda(t-s)}|J(s)|_{2} d s\right)
$$


Choosing $\alpha<\omega$, we define $D_{1}=\left\{(x, v)|| v_{1}|+| v_{2} \mid>M_{\alpha}\right\}$ and $D_{2}=D_{1}^{c}$ where $M_{\alpha}$ is as in Lemma 3.2. We also denote $F=\left|E_{1}\right|+\left|E_{2}\right|+|B|$. Then

$$
\begin{aligned}
& \left|\int_{0}^{P} E_{1, n}(t) d x\right| \leq C \int_{0}^{t} \int_{0}^{s} \iint_{\mathbb{R}_{P} \times \mathbb{R}^{2}}\left\{|J(s-u)|_{2}\right. \\
& \left.+C_{\Lambda} \int_{0}^{s-u} e^{\Lambda\left(s-u-u_{1}\right)}\left|J\left(u_{1}\right)\right|_{2} d u_{1}\right\}|F f|(u) d x d v d u d s \\
& =C \int_{0}^{t} \int_{0}^{s} \iint_{D_{1}} C_{\alpha}^{1} e^{\alpha(s-u)}|F(u)|_{\infty}|f(u)| d x d v d u d s \\
& +C \int_{0}^{t} \int_{0}^{s} \iint_{D_{2}}|J(s-u)|_{2} d x d v|F(u)|_{\infty}|f(u)|_{\infty} d u d s \\
& +C C_{\Lambda} \int_{0}^{t} \int_{0}^{s} \int_{0}^{s-u} e^{\Lambda\left(s-u-u_{1}\right)} \iint_{D_{1}} C_{\alpha}^{1} e^{\alpha u_{1}}|F(u)|_{\infty}|f(u)| d x d v d u_{1} d u d s \\
& +C C_{\Lambda} \int_{0}^{t} \int_{0}^{s} \int_{0}^{s-u} e^{\Lambda\left(s-u-u_{1}\right)} \iint_{D_{2}}\left|J\left(u_{1}\right)\right|_{2} d x d v d u_{1}|F(u)|_{\infty}|f(u)|_{\infty} d u d s \\
& =I+I I+I I I+I V \text {, }
\end{aligned}
$$

By Lemma 3.9 and Lemma 3.2,

$$
\begin{aligned}
I & \leq C \int_{0}^{t} \int_{0}^{s} C_{\alpha}^{1} e^{\alpha(s-u)}\left(D \delta e^{\omega u}\right)^{2} d u d s \leq C^{\prime}\left(\delta e^{\omega t}\right)^{2} \\
I I & \leq C \int_{0}^{t} \int_{0}^{s} C_{\alpha}^{2} e^{\alpha(s-u)}\left(D \delta e^{\omega u}\right)^{2} d u d s \leq C^{\prime \prime}\left(\delta e^{\omega t}\right)^{2} \\
I I I & \leq C C_{\Lambda} \int_{0}^{t} \int_{0}^{s} \int_{0}^{s-u} e^{\Lambda\left(s-u-u_{1}\right)} C_{\alpha}^{1} e^{\alpha u_{1}}\left(D \delta e^{\omega u}\right)^{2} d u_{1} d u d s \\
& \leq C^{\prime \prime \prime}\left(\delta e^{\omega t}\right)^{2} \\
I V & \leq C C_{\Lambda} \int_{0}^{t} \int_{0}^{s} \int_{0}^{s-u} e^{\Lambda\left(s-u-u_{1}\right)} C_{\alpha}^{2} e^{\alpha u_{1}}\left(D \delta e^{\omega u}\right)^{2} d u_{1} d u d s \\
& \leq C^{i v}\left(\delta e^{\omega t}\right)^{2} .
\end{aligned}
$$

Combining these estimates, we have

$$
\left|\int_{0}^{P} E_{1, n}(t) d x\right| \leq C_{1}\left(\delta e^{\omega t}\right)^{2}
$$

for $t \leq T^{*}=\frac{1}{\omega} \ln \frac{\theta}{\delta}$, where $\theta$ is as in Lemma 3.9.

Now we estimate the second term in (3.37). As above,

$$
\begin{aligned}
& \int_{0}^{P} \partial_{x} E_{1, n}(t) a(x) d x=\iint f_{n}(t, x, v) a(x) d v d x \\
= & \int_{0}^{t} \int_{0}^{s} \iint \nabla_{v} g_{2}(s-u, x, v) \cdot\left(\left(E_{1}+\hat{v}_{2} B\right) f,\left(E_{1}-\hat{v}_{1} B\right) f\right)(u) d v d x d u d s
\end{aligned}
$$


where $g_{1}(t, x, v)$ is the first component of $e^{t \mathscr{L}^{*}}[a(x), 0,0,0]$. Since $|a|_{W^{1, \infty}} \leq 1$, by Lemma 3.8 (III) we still have

$$
\left|\nabla v g_{2}(t, x, v)\right| \leq C\left(|J(t)|_{2}+C_{\Lambda} \int_{0}^{t} e^{\Lambda(t-s)}|J(s)|_{2} d s\right)
$$

and we can estimate the term in (3.38) in the same way as above. Combining these estimates, we have $\left\|E_{1, n}(t)\right\|_{1} \leq C_{2}\left(\delta e^{\omega t}\right)^{2}$ for some $C_{2}>0$ as $t \leq T^{*}$. By Lemma 3.4 , we have

$$
\left|E_{2, n}\right|_{1}(t) \leq\left|\int_{0}^{P} E_{2, n}(t) d x\right|+2 \sup _{\substack{a \in W_{\text {per }}^{1, \infty}(0, P) \\\left\|a_{x}\right\|_{\infty} \leq 1}} \int_{0}^{P} \partial_{x} E_{2, n}(t) a(x) d x
$$

and

$$
\left|B_{n}\right|_{1}(t) \leq\left|\int_{0}^{P} B_{n}(t) d x\right|+2 \sup _{\substack{a \in W_{\text {per }}^{1, \infty}(0, P) \\\left\|a_{x}\right\|_{\infty} \leq 1}} \int_{0}^{P} \partial_{x} B_{n}(t) a(x) d x
$$

By the same method,

$$
\left|\int_{0}^{P} E_{2, n}(t) d x\right|=\left|\int_{0}^{t} \iint_{\mathbb{R}_{P} \times \mathbb{R}^{2}} \hat{v}_{2} f_{n}(s) d v d x d s\right| \leq C_{3}\left(\delta e^{\omega t}\right)^{2}
$$

for some $C_{3}>0$ and

$$
\int_{0}^{P} B_{n}(t) d x=\int_{0}^{P} B_{n}(0) d x=0
$$

Thus we only need to estimate the second term on the right side of (3.39) and (3.40).

To accomplish this, we shall use the field representation formulae in Section 3.2. A typical term in the expression of $\partial_{x} E_{2, n}$ and $\partial_{x} B_{n}$ has the form $I^{n}(t, x)=$ $I_{0}^{n}+I_{1}^{n}+I_{2}^{n}+I_{3}^{n}$ as in (3.10). We estimate each term as follows. First

$$
\begin{aligned}
& \left|\int_{0}^{P} I_{3}^{n}(t, x) a(x) d x\right| \\
& \leq C \int_{0}^{t}|F(\tau)|_{\infty}\|\langle v\rangle f(\tau)\|_{1} d \tau \leq C \int_{0}^{t}\left(D \delta e^{\omega \tau}\right)^{2} d \tau \leq C_{4}\left(\delta e^{\omega \tau}\right)^{2}
\end{aligned}
$$


for some $C_{4}>0$, by Lemma 3.9. For $I_{2}^{n}$, we have

$$
\begin{aligned}
& \int_{0}^{P} I_{2}^{n}(t, x) a(x) d x \\
& =\int_{0}^{t}\left(\left(\begin{array}{c}
0 \\
h_{1}(x) \\
h_{2}(x) \\
h_{3}(x)
\end{array}\right) a(x+t-\tau),\left(\begin{array}{c}
0 \\
E_{1, n} \\
E_{2, n} \\
B_{n}
\end{array}\right)(\tau, x)\right) d \tau \\
& =\int_{0}^{t}\left(\left(\begin{array}{c}
0 \\
h_{1} \\
h_{2} \\
h_{3}
\end{array}\right) a(x+t-\tau), \int_{0}^{\tau} e^{(\tau-s) \mathscr{L}}\left(\begin{array}{c}
-\nabla_{v} \cdot\left(\left(E_{1}+\hat{v}_{2} B\right) f,\left(E_{1}-\hat{v}_{1} B\right) f\right)(s) \\
0 \\
0 \\
0
\end{array}\right) d s\right) d \tau \\
& =\int_{0}^{t} \int_{0}^{\tau} \iint \nabla_{v} g_{3}^{t-\tau}(\tau-s, x, v) \cdot\left(\left(E_{1}+\hat{v}_{2} B\right) f,\left(E_{1}-\hat{v}_{1} B\right) f\right)(s) d x d v d s d \tau
\end{aligned}
$$

where

$$
\begin{gathered}
h_{1}(x)=\int \partial_{v_{1}}\left(\frac{\hat{v}_{2}}{1-\hat{v}_{1}}\right) f^{0} d v \\
h_{2}(x)=\int \partial_{v_{2}}\left(\frac{\hat{v}_{2}}{1-\hat{v}_{1}}\right) f^{0} d v \\
h_{3}(x)=\int\left(\partial_{v_{1}}\left(\frac{\hat{v}_{2}}{1-\hat{v}_{1}}\right) \hat{v}_{2}-\partial_{v_{2}}\left(\frac{\hat{v}_{2}}{1-\hat{v}_{1}}\right) \hat{v}_{1}\right) f^{0} d v
\end{gathered}
$$

and $g_{3}^{t-\tau}(\tau-s, x, v)$ is the first component of

$$
e^{(\tau-s) \mathscr{L}^{*}}\left(\begin{array}{c}
0 \\
h_{1}(x) a(x+t-\tau) \\
h_{2}(x) a(x+t-\tau) \\
h_{3}(x) a(x+t-\tau)
\end{array}\right)=e^{(\tau-s) \mathscr{L}^{*}} w^{t-\tau} .
$$

Since $h_{i}(x)$ is differentiable and $\|a\|_{W^{1, \infty}} \leq P$, we have $\left\|w^{t-\tau}\right\|_{W^{1, \infty}} \leq C$ (with a constant independent of $t, \tau)$. So by Lemma 3.8 (III),

$$
\left|\partial_{v} g_{3}^{t-\tau}(\tau-s, x, v)\right| \leq C\left(|J(\tau-s)|_{2}+C_{\Lambda} \int_{0}^{\tau-s} e^{\Lambda(\tau-s-u)}|J(u)|_{2} d u\right) .
$$

Moreover,

$$
\begin{aligned}
& \left|\int_{0}^{P} I_{2}^{n}(t, x) a(x) d x\right| \leq C \int_{0}^{t} \int_{0}^{\tau} \iint_{\mathbb{R}_{P} \times \mathbb{R}^{2}} \\
& C\left(|J(\tau-s)|_{2}+C_{\Lambda} \int_{0}^{\tau-s} e^{\Lambda(\tau-s-u)}|J(u)|_{2} d u\right)|F(s)||f(s)| d x d v d s d \tau \\
& \leq C_{5}\left(\delta e^{\omega \tau}\right)^{2}
\end{aligned}
$$


for some $C_{5}>0$ as $t \leq T^{*}$, by using the same calculations as in the estimate of $\left|\int_{0}^{P} E_{1, n}(t) d x\right|$. Now we estimate

$$
\begin{aligned}
& \int_{0}^{P} I_{1}^{n}(t, x) a(x) d x \\
= & \int_{0}^{t} \iint a(x+t-\tau) G(x, v) f_{n}(\tau, x, v) d x d v d \tau \\
= & \int_{0}^{t} \int_{0}^{\tau} \iint \nabla v g_{4}^{t-\tau}(\tau-s, x, v) \cdot\left(\left(E_{1}+\hat{v}_{2} B\right) f,\left(E_{1}-\hat{v}_{1} B\right) f\right)(s) d x d v d s d \tau,
\end{aligned}
$$

where

$$
G(x, v)=\nabla v\left(\frac{\hat{v}_{2}}{1-\hat{v}_{1}}\right) \cdot\left(\left(E_{1}^{0}+\hat{v}_{2} B^{0}\right),-\hat{v}_{1} B^{0}\right)
$$

and $g_{4}^{t-\tau}(\tau-s, x, v)$ is the first component of

$$
e^{(\tau-s) \mathscr{L}^{*}}\left(\begin{array}{c}
a(x+t-\tau) G(x, v) \\
0 \\
0 \\
0
\end{array}\right)=e^{(\tau-s) \mathscr{L}^{*}}\left(\begin{array}{c}
G^{t-\tau}(x, v) \\
0 \\
0 \\
0
\end{array}\right) \text {. }
$$

Since by simple calculations $\left|\nabla_{v}^{2}\left(\frac{\hat{v}_{2}}{1-\hat{v}_{1}}\right) f_{0}\right| \leq C\langle v\rangle^{2}$ and $\|a\|_{W^{1, \infty}} \leq P$, we have $G^{t-\tau} \in L^{\infty, 1}\left(\mathbb{R}_{P} \times \mathbb{R}^{2}\right), \partial_{\nu} G^{t-\tau} \in L^{\infty, 2}\left(\mathbb{R}_{P} \times \mathbb{R}^{2}\right)$ and

$$
\left.\left\|G^{t-\tau}\right\|_{L^{\infty, 1}}+\left\|\partial_{\nu} G^{t-\tau}\right\|_{L^{\infty, 2}} \leq C \text { (independent of } t, \tau\right) \text {. }
$$

Thus by Lemma 3.8 (IV) we have

$$
\left|\partial_{v} g_{4}^{t-\tau}(\tau-s, x, v)\right| \leq C\langle v\rangle^{2}|J(\tau-s)|_{2}+C_{\Lambda} \int_{0}^{\tau-s} e^{\Lambda(\tau-s-u)}|J(u)|_{2} d u
$$

and

$$
\begin{aligned}
& \left|\int_{0}^{P} I_{1}^{n}(t, x) a(x) d x\right| \\
\leq & C \int_{0}^{t} \int_{0}^{\tau} \iint\langle v\rangle^{2}|J(\tau-s)|_{2}|F(s)||f(s)| d x d v d s d \tau \\
+ & C_{\Lambda} \int_{0}^{t} \int_{0}^{\tau} \iiint_{0}^{\tau-s} e^{\Lambda(\tau-s-u)}|J(u)|_{2} d u|F(s)||f(s)| d x d v d s d \tau \\
= & I+I I .
\end{aligned}
$$


The second term is handled in the same way as above to deduce $I I \leq C_{5}\left(\delta e^{\omega \tau}\right)^{2}$. As for the first term, we use Lemmas 3.9 and 3.2 to obtain

$$
\begin{aligned}
I & \leq C \int_{0}^{t} \int_{0}^{\tau} \iint_{D_{1}} C_{\alpha}^{1} e^{\alpha(\tau-s)}|F(s)|_{\infty}\langle v\rangle^{2}|f(s)| d x d v d s d \tau \\
& +C_{\alpha} \int_{0}^{t} \int_{0}^{\tau} \iint_{D_{1}}|J(\tau-s)|_{2}|F(s)|_{\infty}|f(s)|_{\infty} d x d v d s d \tau \\
& \leq C_{\alpha} \int_{0}^{t} \int_{0}^{\tau} e^{\alpha(\tau-s)}\left(D \delta e^{\omega s}\right)^{2} d s d \tau \leq C_{6}\left(\delta e^{\omega \tau}\right)^{2} .
\end{aligned}
$$

Combining these estimates, we deduce

$$
\left|\int_{0}^{P} I_{1}^{n}(t, x) a(x) d x\right| \leq C_{7}\left(\delta e^{\omega \tau}\right)^{2}
$$

for $t \leq T^{*}$. The estimate of the first term $I_{0}^{n}$ is similar to the estimate for $I_{1}^{n}$ and we have

$$
\left|\int_{0}^{P} I_{0}^{n}(t, x) a(x) d x\right| \leq C_{8}\left(\delta e^{\omega \tau}\right)^{2}
$$

for $t \leq T^{*}$. Combining all of the preceding estimates, we conclude that

$$
\left\|E_{1, n}(t)\right\|_{1}+\left\|E_{2, n}(t)\right\|_{1}+\left\|B_{n}(t)\right\|_{1} \leq c_{3}\left(\delta e^{\omega t}\right)^{2}
$$

for some $c_{3}>0$ as $t \leq T^{*}$. This completes the nonlinear instability proof.

Acknowledgment. The work was supported in part by the NSF under Grants DMS-0405066 and DMS-0505460.

\section{Bibliography}

[1] Arnold, V. I., On conditions for non-linear stability of plane stationary curvilinear flows of an ideal fluid. Dokl. Akad. Nauk SSSR 162 (1965), 975-978.

[2] Bernstein, Ira B., Waves in a Plasma in a Magnetic Field, Phys. Rev. 109 (1958), 10-21.

[3] Gardner, C. S., Bound on the energy available from a plasma. Phys. Fluids 6 (1963), 839-840.

[4] Glassey, R. and Strauss, W., Singularity formation in a collisionless plasma could occur only at high velocities. Arch. Rational Mech. Anal. 92 (1986), 59-90.

[5] Glassey, R. and Schaeffer, J., On the "one and one-half dimensional” relativistic VlasovMaxwell system. Math. Methods Appl. Sci. 13 (1990), 169-179.

[6] Guo, Y., Stable magnetic equilibria in collisionless plasma, Comm. Pure Appl. Math. 50, 0891-0933 (1997).

[7] Guo, Y., Strauss, W., Instability of periodic BGK equilibria, Comm. Pure Appl. Math. 48, 861894 (1995).

[8] Guo, Y., Strauss, W., Unstable oscillatory-tail waves in collisionless plasmas, SIAM J. Math. Anal. 30, 1076-1114 (1999).

[9] Guo, Y., Strauss, W., Magnetically created instability in a collisionless plasma, J. Math. Pures Appl. 79, , 975-1009 (2000).

[10] Holm, D., Marsden, J., Ratiu, T. and Weinstein, A., Nonlinear stability of fluid and plasma equilibria. Phys. Rep. 123 (1985), no. 1-2, 116 p.

[11] Lin, Zhiwu, Some stability and instability criteria for ideal plane flows, Comm. Math. Phys., 246, 87-112(2004). 
[12] Lin, Zhiwu, Nonlinear instability of periodic waves for Vlasov-Poisson system, Comm. Pure. Appl. Math. 58, 505-528 (2005).

[13] Lin, Zhiwu, Nonlinear instability of ideal plane flows, Int. Math. Res. Not. 41, 2147-2178 (2004).

[14] Lin, Zhiwu, A simplified proof of the Wolansky-Ghil Theorem, unpublished notes.

[15] Lin, Zhiwu and Strauss, W., Linear stability and instability of relativistic Vlasov-Maxwell systems, to appear in Comm. Pure. Appl. Math.

[16] Wolansky, G. and Ghil, M., Nonlinear stability for saddle solutions of ideal flows and symmetry breaking, Comm. Math. Phys. 193, 713-736(1998).

Received Month 200X. 